\title{
Tissue MicroRNA profiles as diagnostic and prognostic biomarkers in patients with resectable pancreatic ductal adenocarcinoma and periampullary cancers
}

Dan Calatayud ${ }^{1,11^{*}}$ (D), Christian Dehlendorff, Mogens K. Boisen ${ }^{3}$, Jane Preuss Hasselby ${ }^{4}$, Nicolai Aagaard Schultz ${ }^{1}$ Jens Werner ${ }^{5}$, Heike Immervol ${ }^{6,7}$, Anders Molven ${ }^{6,8}$, Carsten Palnæs Hansen ${ }^{1}$ and Julia S. Johansen ${ }^{3,9,10}$

\begin{abstract}
Background: The aim of this study was to validate previously described diagnostic and prognostic microRNA expression profiles in tissue samples from patients with pancreatic cancer and other periampullary cancers.

Methods: Expression of 46 selected microRNAs was studied in formalin-fixed paraffin-embedded tissue from patients with resected pancreatic ductal adenocarcinoma $(n=165)$, ampullary cancer $(n=59)$, duodenal cancer $(n=6)$, distal common bile duct cancer $(n=21)$, and gastric cancer $(n=20)$; chronic pancreatitis $(n=39)$; and normal pancreas $(n=35)$. The microRNAs were analyzed by PCR using the Fluidigm platform.

Results: Twenty-two microRNAs were significantly differently expressed in patients with pancreatic cancer when compared to healthy controls and chronic pancreatitis patients; 17 miRNAs were upregulated (miR-21$5 p,-23 a-3 p,-31-5 p,-34 c-5 p,-93-3 p,-135 b-3 p,-155-5 p,-186-5 p,-196 b-5 p,-203,-205-5 p,-210,-222-3 p$, $-451,-492,-614$, and miR-622) and 5 were downregulated (miR-122-5p, -130b-3p, -216b, -217, and miR375). MicroRNAs were grouped into diagnostic indices of varying complexity. Ten microRNAs associated with prognosis were identified (let-7 g, miR-29a-5p, -34a-5p, -125a-3p, -146a-5p, -187, -205-5p, -212-3p, -222$5 p$, and miR-450b-5p). Prognostic indices based on differences in expression of 2 different microRNAs were constructed for pancreatic and ampullary cancer combined and separately (30, 5, and 21 indices).

Conclusion: The study confirms that pancreatic cancer tissue has a microRNA expression profile that is different from that of other periampullary cancers, chronic pancreatitis, and normal pancreas. We identified prognostic microRNAs and microRNA indices that were associated with shorter overall survival in patients with radically resected pancreatic cancer.
\end{abstract}

Keywords: Ampullary cancer, Biomarkers, microRNA, Pancreatic ductal adenocarcinoma, Pancreatic cancer

\section{Background}

Pancreatic cancer (PC) is the fourth most common cause of cancer-related death in the Western world, although it only represents $3 \%$ of all new cancer cases $[1,2]$. Most cases are pancreatic ductal adenocarcinomas (PDAC). Due to locally advanced or metastatic disease, only $20 \%$ of

\footnotetext{
* Correspondence: dan.calatayud@gmail.com

'Department of Surgical Gastroenterology and Transplantation,

Rigshospitalet, Copenhagen University Hospital, Copenhagen, Denmark

${ }^{11}$ Department of Oncology, Herlev University Hospital, Herlev Ringvej 75,

DK-2730 Herlev, Denmark

Full list of author information is available at the end of the article
}

all patients diagnosed with PC are accessible to radical surgical treatment, and thereby have the potential for long-term survival $[3,4]$. However, even in this group, the 5 -year survival is only $20 \%$ due to the high recurrence rate $[5,6]$.

PC located in the head of the pancreas constitutes the majority $(60-70 \%)$ of the group of cancers in the region, which also includes of ampullary adenocarcinomas (A-AC), accounting for 15-25\%; and duodenal cancers (DC); and distal common bile duct (CBD) cancers, each accounting for approximately $10 \%[6]$. 
The distribution of the different types of the periampullary cancers is variously reported, probably due to the complexity of the periampullary anatomy and histopathology. The 5-year survival rate after surgery is $45-55 \%$ for $\mathrm{A}-\mathrm{AC}$ and $\mathrm{DC}[7,8]$ and approximately $25 \%$ for distal CBD cancers [6].

Cancer antigen 19-9 (CA 19-9, also named carbohydrate antigen 19-9 and sialylated Lewis antigen) is the most widely used biomarker for patients with PC. Serum CA19-9 alone is insufficient as a diagnostic biomarker, although it may have prognostic value in the absence of cholestasis [9]. There is an obvious need for better biomarkers in PC, and microRNAs (miRNAs, miRs) could be interesting in this regard.

MiRNAs are small (18-24 nucleotides) non-coding RNAs that regulate gene expression post-transcriptionally by binding to messenger RNA molecules through nucleotide complementarity $[10,11]$. MiRNAs regulate critical cellular processes such as differentiation, proliferation, apoptosis, and metastasis [12-16]. MiRNAs are stable and analyzable in formalin-fixed paraffin-embedded (FFPE) tissue, which is suitable for analysis [17, 18]. So far, 2603human miRNA sequences have been discovered and the number is increasing [19].

The expression patterns of miRNAs can be combined into profiles that are specific for a given type of tissue or disease. Several specific miRNA expression profiles in PC tissue have been described, with a promising consistency between studies and different array or PCR platforms. The expressions of miR-15b, $-21,-95,-103$, $-107,-122,-135 \mathrm{~b},-148 \mathrm{a},-155,-190,-196 \mathrm{a},-200$, $-203,-210,-216 \mathrm{~b},-217,-221,-222$, and miR-375 differ between PC and normal pancreas or chronic pancreatitis [20-28]. Furthermore, miRNA expression profiling indicates a close relationship between PDAC and A-AC [27]. Specific miRNAs have also been suggested as prognostic biomarkers in several cancers, including PC [23, 29-32].

The aim of the present study was to validate previously described diagnostic and prognostic miRNA expression profiles for PDAC and A-AC in FFPE specimens.

\section{Methods}

\section{Patients}

\section{Diagnostic miRNA study}

FFPE tumor specimens $(n=359$ including an internal control) were obtained from patients who underwent resection with radical intent for the following diagnoses: PDAC $(n=165)$, A-AC $(n=59), \mathrm{DC}(n=6)$, distal CBD cancer $(n=21)$, chronic pancreatitis (CP) $(n=39)$, gastric cancer $(\mathrm{GC})(n=20)$, serous cyst adenoma $(n=2)$, and no cancer $(n=4$; cysts or fibrosis that could not be classified as normal pancreas or pancreatitis and did not have any malignant foci) and healthy subjects (HS) $(n=35)$. The pancreatic and periampullary specimens came from patients who had undergone pancreaticoduodenectomy, distal pancreatectomy, or total pancreatectomy between 2004 and 2011 in Denmark (Herlev Hospital $n=$ 9; Rigshospitalet $n=198$ ), Germany (Heidelberg $n=69$ ), and Norway (Bergen $n=55$ ). The chronic pancreatitis specimens came from Copenhagen $(n=5)$ and Heidelberg $(n=34)$. All normal pancreas tissue was obtained from Heidelberg from organ donors or patients with traumatic pancreatic lesions leading to resection of healthy pancreatic tissue. The Danish patients were included in the BIOPAC Study (BIOmarkers in patients with Pancreatic Cancer). The gastric cancers came from patients who had undergone surgery at Gentofte Hospital. An experienced pathologist reassessed all samples to select the most representative part of the specimen, and tumors were classified and graded according to the World Health Organization criteria [33].

\section{Prognostic miRNA study}

One hundred fifty-seven FFPE tumor specimens were analyzed from patients who underwent surgery with radical intent for PDAC $(n=103)$ and A-AC $(n=54)$. The patients were included in the BIOPAC Study at Rigshospitalet in Denmark. Inclusion criteria were age $\geq 18$ years and histologically verified $\mathrm{PC}$ in a resected specimen. After surgery, the majority of the patients $(87 \%)$ were treated with adjuvant gemcitabine for 6 months or until disease recurrence.

Patient characteristics are shown in Table 1.

\section{MiRNA purification from FFPE tissues}

One FFPE block was selected from each patient for miRNA analysis. From each of these blocks, $310-\mu \mathrm{m}$ sections were cut for miRNA extraction without microdissection. As method control, $9 \times 3$ sections were cut from a specimen from 1 of the PDAC patients. MiRNAs were extracted using Qiagen miRNeasy FFPE kit, Cat No./ID: 217504. Briefly, the sections were deparaffinized in xylene and ethanol and then treated with proteinase $\mathrm{K}$, and RNA was isolated using the one-column spin column protocol for total RNA. The concentration of small RNAs was assessed by absorbance spectrometry on a DTX 880 (Beckman Coulter).

\section{MiRNA analysis}

The following 46 miRNAs were selected for analysis: miR-21-5p, -23a-3p, -29a-5p, -31-5p, -34a-5p, -34c5p, -93-3p, -122-5p, -125a-3p, -130b-3p, -135b-3p, -136-3p, -146a-5p, -148a-3p, -148a-5p, -155-5p, -1865p, -187-3p, -194-3p, -196b-5p, -198, -203, -205-5p, $-210,-212-3 p,-216 b,-217,-222-3 p,-222-5 p,-375$, $-411-5 p,-431-5 p,-450 b-5 p,-451 a,-490-3 p,-492$, 
Table 1 Characteristics of the Danish patients

\begin{tabular}{|c|c|c|c|c|c|c|}
\hline Characteristic & $\begin{array}{l}\text { PDAC } \\
N=110\end{array}$ & $\begin{array}{l}A-A C \\
N=59\end{array}$ & $\begin{array}{l}\text { Duodenal cancer } \\
\mathrm{N}=6\end{array}$ & $\begin{array}{l}\text { Distal CBD cancer } \\
N=21\end{array}$ & $\begin{array}{l}\text { Chronic pancreatitis } \\
\mathrm{N}=5\end{array}$ & $\begin{array}{l}\text { Serous cystadenoma and } \\
\text { other benign diagnosis } \\
N=6\end{array}$ \\
\hline Age, years median (range) & $65.7(37.4-81.3)$ & $64.9(38.3-80.5)$ & $69.0(54.3-74.4)$ & $64.7(38.6-74.6)$ & $56.4(43.8-68.2)$ & $60.6(46.7-84.7)$ \\
\hline \multicolumn{7}{|l|}{ Gender } \\
\hline Male & $60(55 \%)$ & $37(63 \%)$ & $5(83 \%)$ & $11(52 \%)$ & $5(100 \%)$ & $2(33 \%)$ \\
\hline Female & $50(45 \%)$ & $22(37 \%)$ & $1(17 \%)$ & $10(48 \%)$ & 0 & $4(67 \%)$ \\
\hline \multicolumn{7}{|l|}{ ASA score } \\
\hline 1 & $12(11 \%)$ & $9(15 \%)$ & 0 & $2(10 \%)$ & $1(20 \%)$ & 0 \\
\hline 2 & $58(53 \%)$ & $38(66 \%)$ & $5(83 \%)$ & $15(75 \%)$ & $2(40 \%)$ & $4(80 \%)$ \\
\hline 3 & $30(27 \%)$ & $11(19 \%)$ & $1(17 \%)$ & $3(15 \%)$ & $2(40 \%)$ & $1(20 \%)$ \\
\hline 4 & 0 & 0 & & 0 & 0 & 0 \\
\hline \multicolumn{7}{|l|}{ TNM-Stage } \\
\hline IA & $9(8 \%)$ & $4(7 \%)$ & $1(17 \%)$ & $1(5 \%)$ & & \\
\hline IB & $3(3 \%)$ & $7(12 \%)$ & $1(17 \%)$ & $1(5 \%)$ & & \\
\hline$\| \mathrm{A}$ & $27(25 \%)$ & $6(10 \%)$ & $2(33 \%)$ & $7(52 \%)$ & & \\
\hline$\| \mathrm{B}$ & $67(65 \%)$ & $24(41 \%)$ & $2(33 \%)$ & $11(33 \%)$ & & \\
\hline III & 0 & $16(27 \%)$ & 0 & $1(5 \%)$ & & \\
\hline
\end{tabular}

Values are $\mathrm{N}$ (\%). Numbers may not add up due to missing values

No clinical information is available from the patients with gastric cancer and the patients and healthy subjects from Heidelberg and Bergen

$-509-5 \mathrm{p},-571,-614,-622,-625-5 \mathrm{p},-675-5 \mathrm{p},-769-5 \mathrm{p}$, $-939,-944$, and let-7 g. The selection was based on the previously described relationship of the miRNAs to PC in particular and to cancer biology in general (Detailed information on each specific miRNA is available in "Additional file 1").

The miRNAs were analyzed in triplicate using the Fluidigm BioMark System ${ }^{\mathrm{rm}}$. This system can perform multiple simultaneous real-time PCR measurements running gold-standard Taqman $^{\odot}$ assays in nanolitre quantities. The instructions from Fluidigm were followed in all details (https://www.fluidigm.com). The analyses were performed at AROS Applied Biotechnology A/S (www.arosab.com, Aarhus, Denmark).

\section{Statistical analysis}

Differences in miRNA expression according to diagnosis were tested by univariate logistic regression including the raw miRNA expression level as continuous variables on the cycle threshold scale. Odds ratios (OR) per interquartile increase and $95 \%$ confidence intervals were computed for both PC vs. HS and PC vs. HS and CP.

Diagnostic indices were identified in 3 different ways among the significant miRNAs: (1) As a manually defined index by including 2 miRNA with OR $>1$ and 2 with $\mathrm{OR}<1$ (indices I and IV);(2) As a computer generated index found by backwards elimination of a model with miRNAs chosen from 18 miRNAs described in an previous index (the so-called LASSO-classifier: miR-23a, 34c-5p, -122, -135b-3p, -136-3p, -186, -196b, -198,
$-203,-222-3 p,-451,-490,-492,-509-5 p,-571,-614$, -622 , and miR-93 [27]) which were significant at a $1 \%$ significance level, to account for multiple testing and with less than $10 \%$ missing values (indices II and V) and (3) as a computer generated index like (2) but based on all significant miRNAs (indices III and VI). A total of 6 indices were identified: I, II, and III developed for the PC vs. HS comparison and IV, V, and VI developed for the PC vs. HS + CP comparison. The indices were evaluated by means of boxplots, and their performance was evaluated by computing sensitivity, specificity, accuracy, area under curve (AUC), true positives (TP), true negatives (TN), false positives (FP), and false negatives (FN). The indices were also tested on other cancer types. For each index, we first found a suitable cut-off by requiring a sensitivity of $85 \%$ in the PC vs. HS or vs. HS + CP comparison. Subsequently, this cut-off point was applied in all other comparisons.

It was not possible to stratify our patients according to TNM due to the very uneven distribution of cancer stages and resulting small subgroups.

For the prognostic study, the association between overall survival (OS) and miRNA expression was illustrated by Kaplan-Meier curves by dichotomizing the miRNA expression into below and above the median expression for each miRNA. The association was tested by means of univariate Cox proportional hazards regression both on the continuous variables and on the dichotomized variables, and presented as hazard ratios (HR) and corresponding 95\% confidence intervals (CIs). In 
addition, analyses adjusted for age, sex, tumor stage, ASA score, and tumor differentiation were performed. Finally, we considered differences between 2 miRNAs at a time as a continuous variable in the Cox models (unadjusted and adjusted) for OS. Analyses were made for the diagnoses PDAC and A-AC together and separately.

In all analysis, the software package $\mathrm{R}$ version 3.1.1 ( $\mathrm{R}$ Core Team 2014; R: A language and environment for statistical computing. R Foundation for Statistical Computing, Vienna, Austria. www.R-project.org) was used, and $P$-values below $5 \%$ were considered statistically significant.

\section{Results}

\section{Diagnosis - Pancreatic cancer vs. healthy subjects}

The following 14 miRNAs were upregulated in PC compared to HS: miR-21-5p, -23a-3p, -31-5p, -34c-5p, $-93-3 p,-135 b-3 p,-155-5 p,-196 b-5 p,-203,-205-5 p$, $-210,-222-3 p,-451$, and miR-622. The following 5 miRNAs were downregulated in PC: miR-122-5p, -130b-3p, -216b, - 217, and miR-375 (Table 2).

Three indices of miRNA expression, index I, II, and III, were identified to separate PC from HS (i.e., normal pancreas tissue):

(I) A manually defined index: miR-375 + miR-130b-3p miR-451 - miR34c-5p.

(II) A computer-generated index based on univariate significant miRNAs chosen from 18 miRNAs describes in a previous index with less than $10 \%$ missing: $292.6458-$ $3.0539 \times \mathrm{miR}-34 \mathrm{c}-5 \mathrm{p}+4.007 \times \mathrm{miR}-203-10.4 \times \mathrm{miR}-222-3 \mathrm{p}-$ $3.6057 \times \mathrm{miR}-451-4.3015 \times \mathrm{miR}-622$.

The potential miRNAs for index II were miR-34c-5p, -135-3p, -203, -222-3p, -451, and miR-622.

(III) A computer-generated index based on all univariate significant miRNAs with less than $10 \%$ missing values: $118.7249+77.2459 \times \mathrm{miR}-130 \mathrm{~b}-3 \mathrm{p}-23.7911 \times \mathrm{miR}-$ $34 \mathrm{c}-5 \mathrm{p}-49.923 \times \mathrm{miR}-451$.

The potential miRNAs for index III were miR-31-5p, -34c-5p,-93-3p, -130b-3p, -135b-3p, -155-5p, -203, $-205-5 p,-210,-216 b,-217,-222-3 p,-375,-451$, and miR-622.

The performances of these indices are illustrated in box plots in Fig. 1 and Table 3 (upper part). The manually calculated index I was able to separate PC from HS with a sensitivity of 84.9 (CI 78.5-90.0), but could also differentiate the other malignant diagnoses from HS, with a sensitivity varying from 66.7 (distal CBD cancer) to 100.0 (DC and GC). The computer-generated index II performed in the same way with regard to PC vs. HS, but was inferior for separating the other malignancies from HS except for distal CBD cancer, where it performed better than index I. The computer-generated index III performed slightly better than index II with regard to separating $\mathrm{A}-\mathrm{AC}$ and $\mathrm{DC}$ cancer from $\mathrm{HS}$, but was inferior for separating distal CBD cancer and GC.

\section{Diagnosis - Pancreatic cancer vs. healthy subjects + chronic pancreatitis}

The following 17 miRNAs were upregulated in PDAC compared with benign specimens (HS and CP combined): miR-21-5p, -23a-3p, -31-5p, -34c-5p, -93-3p, -135b-3p, -155-5p, -186-5p, -196b-5p, -203, -205-5p, $-210,-222-3 p,-451,-492,-614$, and miR-622. The following 5 miRNAs were downregulated in PDAC compared to benign specimens (HS and CP combined): miR-122-5p, -130b-3p, -216b, -217, and miR375 (Table 2).

Three indices, IV, V, and VI, of miRNA expression to separate PC from benign tissue (i.e., HS and CP combined) were identified.

(IV) A manually defined index: miR-375 + miR-130b$3 p-\operatorname{miR}-451$ - miR-34c-5p.

(V) A computer-generated index based on significant miRNAs chosen from 18 miRNAs described in a previous index with less than $10 \%$ missing values: 20.5487-1.5899 $\times \mathrm{miR}-222-3 \mathrm{p}-0.4006 \times \mathrm{miR}-451-$ $0.3864 \times$ miR-203-0.5056 $\times$ miR-622+ 1.203 $\times$ miR-186-5p.

The potential miRNAs for index $\mathrm{V}$ weremiR-34c-5p, $-135 b-3 p,-186-5 p,-203,-222-3 p,-451$, and miR-622.

(VI) A computer-generated index based on all significant miRNAs with less than $10 \%$ missing values: 7.1834$0.5175 \times$ miR-210 + 1.3893 $\times$ miR-93-3p - 0.7423 $\times$ miR-375$2.6184 \times$ miR-222-3p - 0.3414 $\times$ miR-451-0.3852 $\times$ miR-203$0.5316 \times$ miR- $622+1.822 \times$ miR-186-5p.

The potential miRNAs for index VI were miR-31-5p, -34c-5p, -93-3p, -130b-3p, -135b-3p, -155-5p, -186$5 p,-203,-210,-216 b,-217,-222-3 p,-375,-451$, and miR-622.

The performances of these indices are illustrated in box plots in Fig. 1 and in Table 3 (lower part). Index IV could separate HS from the other diagnoses. Indices V and VI were able to separate $\mathrm{CP}$ from the malignant diagnoses.

\section{Diagnostic miRNA indices previously identified for pancreatic cancer}

We have previously described the following 4 different diagnostic miRNA indices in FFPE cancer tissues consisting of 2 different miRNAs [27]: (1) miR-196b5p - miR-217; (2) miR-411 - miR-198; (3) miR-614 miR-122-5p; and (4) miR-614 - miR-93-3p. The performance of the 4 indices in the present cohort was tested using the Fluidigm method. Since many samples had non-detectable miRNAs, we only used observations that were non-missing, i.e., not imputed by a large $C_{t}$-value. Index 1 had 97 samples with at least 1miRNA missing, index 2 had 122 samples with 
Table 2 Significantly deregulated microRNAs

\begin{tabular}{|c|c|c|c|c|c|}
\hline \multicolumn{6}{|c|}{ microRNA upregulated in PC compared to healthy subjects } \\
\hline miRNA & $\mathrm{OR}(\mathrm{Cl})$ & $p$-value & PC & $\mathrm{HS}$ & Missing \\
\hline miR-21-5p & $0.11(0.03-0.25)$ & 0.0000 & 134 & 13 & 53 \\
\hline miR-23a-3p & $0.36(0.13-0.67)$ & 0.0100 & 156 & 5 & 39 \\
\hline miR-31-5p & $0.38(0.28-0.50)$ & 0.0000 & 165 & 35 & 0 \\
\hline $\operatorname{miR}-34 c-5 p$ & $0.17(0.09-0.28)$ & 0.0000 & 165 & 35 & 0 \\
\hline miR-93-3p & $0.14(0.06-0.26)$ & 0.0000 & 165 & 34 & 1 \\
\hline miR-135b-3p & $0.31(0.20-0.44)$ & 0.0000 & 165 & 30 & 5 \\
\hline miR-155-5p & $0.11(0.03-0.23)$ & 0.0000 & 165 & 33 & 2 \\
\hline miR-196b-5p & $0.14(0.02-0.45)$ & 0.0151 & 147 & 3 & 50 \\
\hline miR-203 & $0.37(0.25-0.51)$ & 0.0000 & 165 & 35 & 0 \\
\hline miR-205-5p & $0.71(0.59-0.82)$ & 0.0000 & 148 & 21 & 31 \\
\hline miR-210 & $0.12(0.05-0.22)$ & 0.0000 & 165 & 34 & 1 \\
\hline miR-222-3p & $0.06(0.02-0.15)$ & 0.0000 & 165 & 35 & 0 \\
\hline miR-451 & $0.14(0.06-0.27)$ & 0.0000 & 165 & 35 & 0 \\
\hline miR-622 & $0.57(0.41-0.76)$ & 0.0003 & 165 & 34 & 1 \\
\hline \multicolumn{6}{|c|}{ microRNA downregulated in PC compared to healthy subjects } \\
\hline miRNA & $\mathrm{OR}(\mathrm{Cl})$ & $p$-value & PC & $\mathrm{HS}$ & Missing \\
\hline miR-122-5p & $2.08(1.40-3.51)$ & 0.0014 & 30 & 18 & 152 \\
\hline miR-130b-3p & $5.34(3.17-9.98)$ & 0.0000 & 165 & 35 & 0 \\
\hline miR-216b & $6.30(3.36-14.24)$ & 0.0000 & 149 & 35 & 16 \\
\hline miR-217 & $2.94(2.03-4.69)$ & 0.0000 & 142 & 35 & 23 \\
\hline miR-375 & $26.10(9.48-90.22)$ & 0.0000 & 165 & 35 & 0 \\
\hline \multicolumn{6}{|c|}{ microRNA upregulated in PC compared to healthy subjects and chronic pancreatitis } \\
\hline miRNA & $\mathrm{OR}(\mathrm{Cl})$ & $p$-value & PC & $\mathrm{HS}+\mathrm{CP}$ & Missing \\
\hline miR-21-5p & $0.24(0.14-0.36)$ & 0.0000 & 134 & 42 & 63 \\
\hline miR-23a-3p & $0.54(0.38-0.74)$ & 0.0003 & 156 & 31 & 52 \\
\hline miR-31-5p & $0.50(0.41-0.59)$ & 0.0000 & 165 & 74 & 0 \\
\hline $\operatorname{miR}-34 c-5 p$ & $0.33(0.25-0.43)$ & 0.0000 & 165 & 74 & 0 \\
\hline miR-93-3p & $0.27(0.17-0.40$ & 0.0000 & 165 & 73 & 1 \\
\hline miR-135b-3p & $0.31(0.22-0.41$ & 0.0000 & 165 & 58 & 16 \\
\hline miR-155-5p & $0.46(0.37-0.56$ & 0.0000 & 165 & 72 & 2 \\
\hline miR-186-5p & $0.71(0.55-0.89$ & 0.0041 & 165 & 74 & 0 \\
\hline miR-196b-5p & $0.53(0.39-0.70$ & 0.0000 & 147 & 20 & 72 \\
\hline miR-203 & $0.36(0.26-0.46$ & 0.0000 & 165 & 74 & 0 \\
\hline miR-205-5p & $0.79(0.71-0.88$ & 0.0000 & 148 & 46 & 45 \\
\hline miR-210 & $0.27(0.18-0.36$ & 0.0000 & 165 & 73 & 1 \\
\hline miR-222-3p & $0.23(0.16-0.32$ & 0.0000 & 165 & 74 & 0 \\
\hline miR-451 & $0.44(0.35-0.54$ & 0.0000 & 165 & 74 & 0 \\
\hline miR-492 & $0.46(0.22-0.78$ & 0.0097 & 57 & 4 & 178 \\
\hline miR-614 & $0.75(0.57-0.94$ & 0.0219 & 110 & 14 & 115 \\
\hline miR-622 & $0.52(0.41-0.66$ & 0.0000 & 165 & 72 & 2 \\
\hline
\end{tabular}


Table 2 Significantly deregulated microRNAs (Continued)

\begin{tabular}{|c|c|c|c|c|c|}
\hline miRNA & $\mathrm{OR}(\mathrm{Cl})$ & $p$-value & $P C$ & $\mathrm{HS}+\mathrm{CP}$ & Missing \\
\hline miR-122-5p & 1.99 (1.46-2.98) & 0.0001 & 30 & 40 & 169 \\
\hline miR-130b-3p & $1.71(1.33-2.23)$ & 0.0001 & 165 & 74 & 0 \\
\hline miR-216b & $1.55(1.34-1.84)$ & 0.0000 & 149 & 73 & 17 \\
\hline miR-217 & $1.46(1.28-1.69)$ & 0.0000 & 142 & 71 & 26 \\
\hline miR-375 & $2.22(1.62-3.15)$ & 0.0000 & 165 & 74 & 0 \\
\hline
\end{tabular}

at least 1 miRNA missing, index 3 had 213 samples with at least 1 miRNA missing, and index 4 had 115 samples with at least 1miRNA missing. For indices 2 and 3 , it was not possible to consider HS alone. The performances of these indices are shown in box plots in Fig. 2. Index 1 could separate HS from PC patients but could not separate CP from A-AC. Index 1 could separate GC from all other diagnoses with high accuracy. Indices 2,3 , and 4 could not separate samples with benign from malignant diagnoses. Further information is given in the "Additional file 2".
Prognostic miRNAs - PDAC and A-AC patients combined In all, 157 patients with either PDAC or A-AC were available for the survival analysis, and 112died during the follow-up period. Table 4 illustrates that low expression of 6 miRNAs (miR-29a-5p, miR-34a-5p, miR-125a3p, miR-146a-5p, miR-205-5p, and miR-212-3p) was associated with short OS, both with and without adjustment for age, sex, tumor stage/differentiation, and ASAscore. When patients were divided into 2 groups for each miRNA (defined as expression under or above the median level), low miR-34a-5p, miR-205-5p, miR-212-

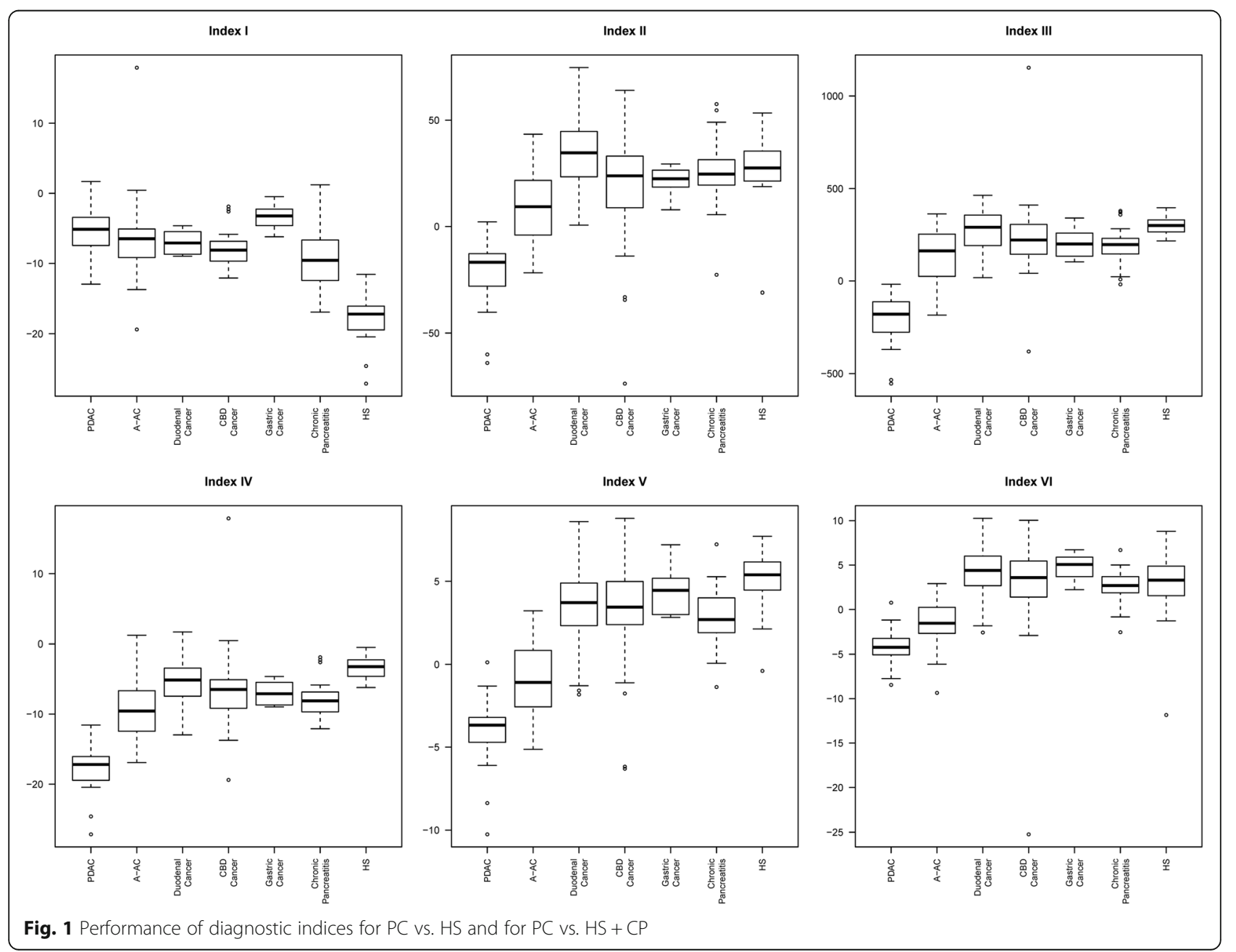




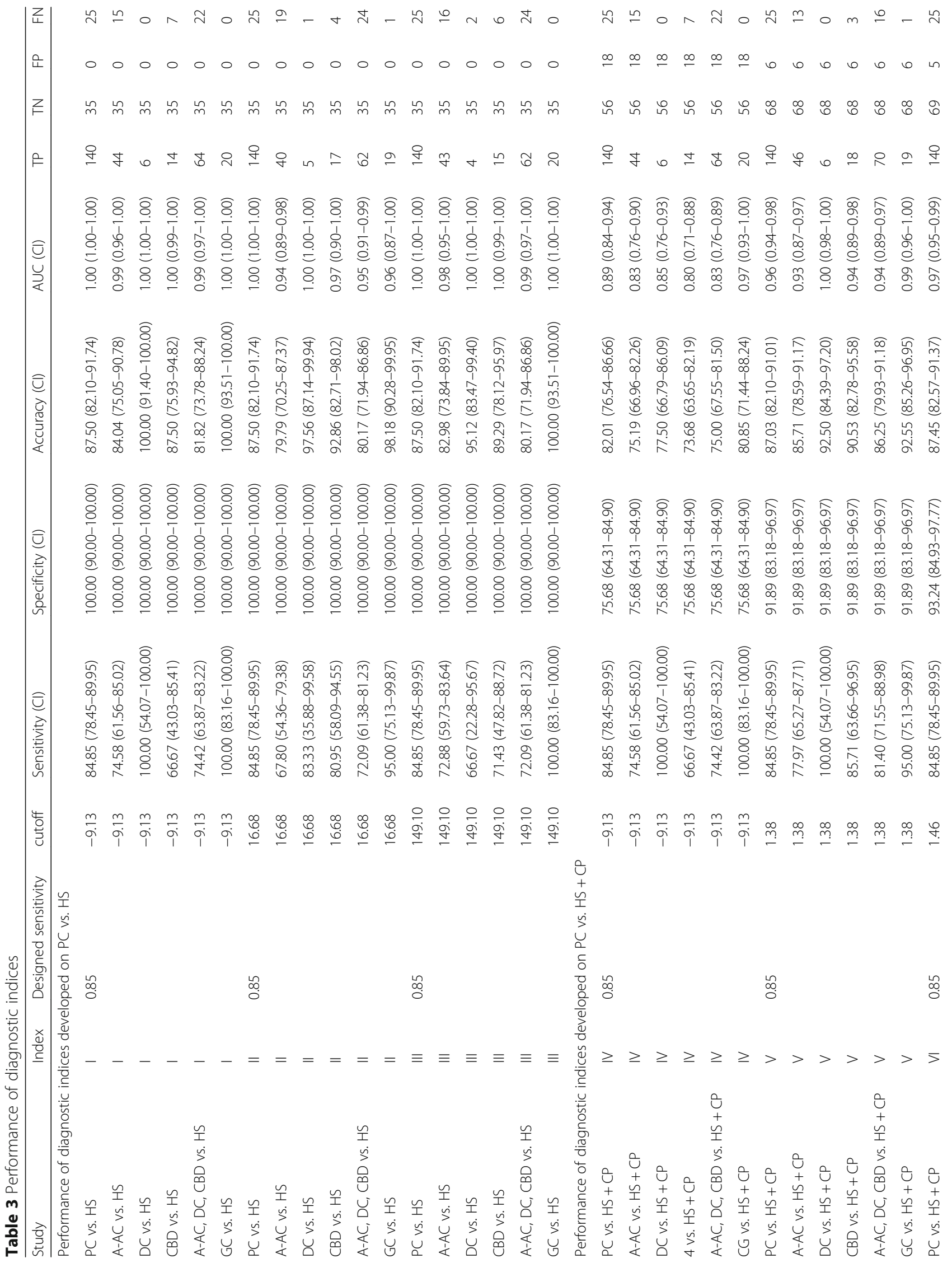




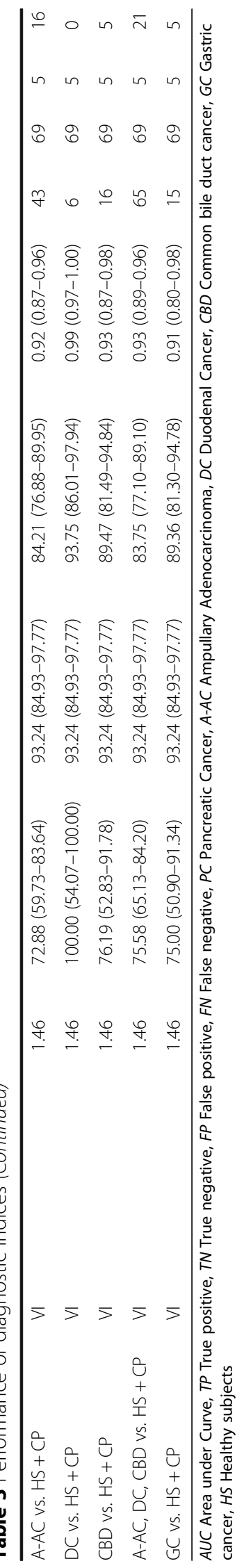



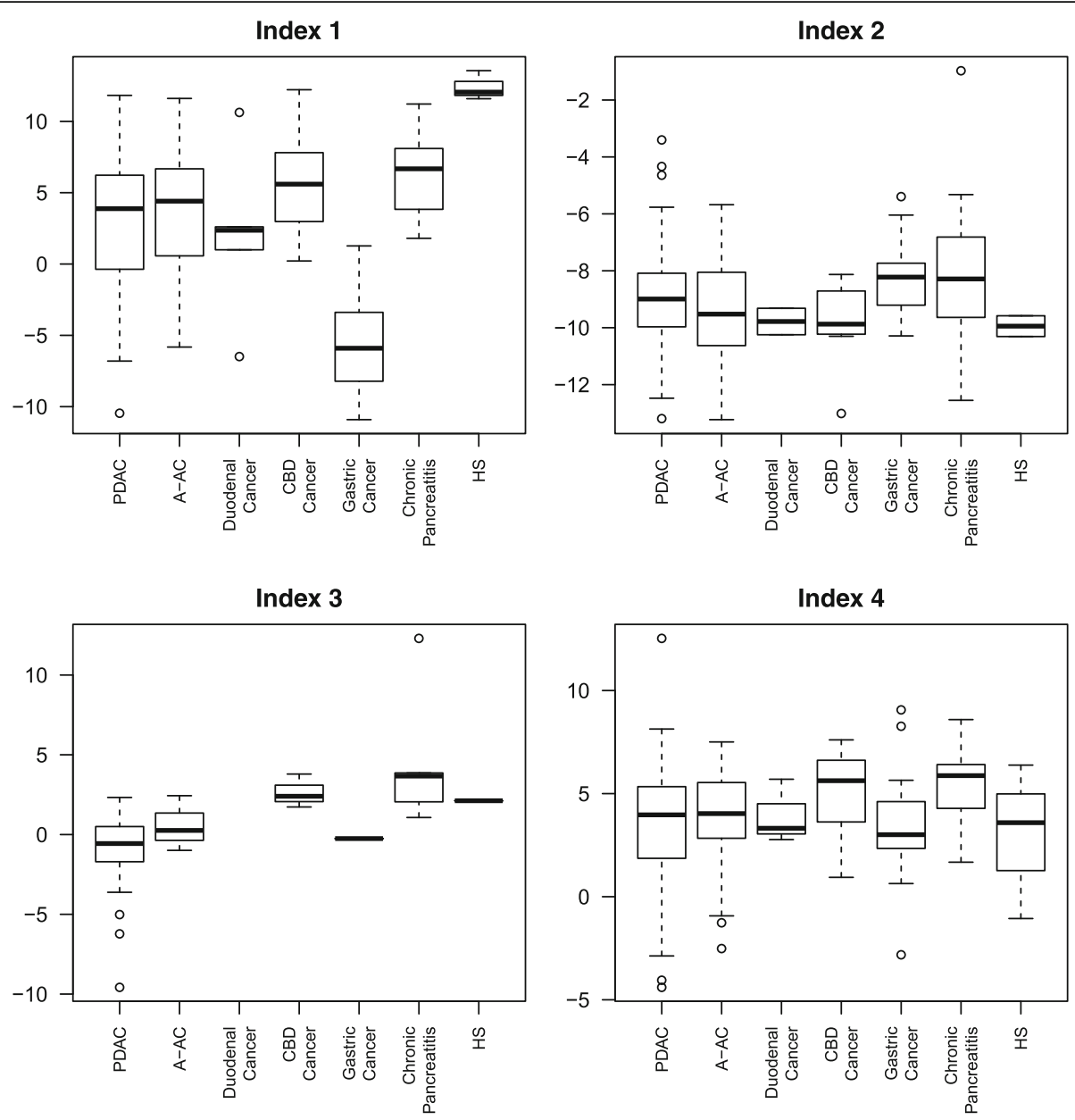

Fig. 2 Performance of two miRNA diagnostic indices

$3 p$, and miR-222-5plevels were significantly associated with short OS. After adjusting for age, sex, tumor stage/ differentiation, and ASA-score, let-7 g, miR-29a-5p, miR-34a-5p, miR-205-5p, and miR-212-3p were associated with short OS. Figure 3 illustrates Kaplan-Meier curves for the 6 miRNAs reaching a significance level below 0.01 .

Table 5 shows 30 and 27 combinations of 2 miRNAs significantly associated with short $O S$ in an unadjusted and an adjusted analysis in PDAC and A-AC in combination.th $=\mathrm{tl} b=$

\section{Prognostic miRNAs - PDAC}

One hundred three patients with PDAC were available for the survival analysis, and 83 died during the followup period. In both the unadjusted and the adjusted (age, sex, tumor stage/differentiation, ASA-score) analyses, low expression of 2 miRNAs was associated with short OS prognosis:miR-34a-5p: $\quad H R=0.72(\mathrm{CI}: \quad 0.56-0.93$ ) (unadjusted) and $\mathrm{HR}=0.70(\mathrm{CI}: 0.52-0.93)$ (adjusted); and miR-212-3p HR $=0.83(\mathrm{CI}: 0.71-0.99)$ (unadjusted) and $\mathrm{HR}=0.82$ (CI: 0.68-0.99) (adjusted). Dividing the patients into 2 groups for each miRNA (defined as expression under or above the median level), low miR34a-5p and miR-212-3p levels were associated with short OS. Figure 4 shows Kaplan-Meier curves for the miRNAs reaching a significance level below 0.01 .

Table 5 shows 5 and 12 combinations of 2 miRNAs significantly associated with short OS in an unadjusted and an adjusted analysis in PDAC.

\section{Prognostic miRNAs - A-AC}

Fifty-four patients with A-AC were available for the survival analysis, and 29 died during the follow-up period. In the unadjusted analysis, 4 miRNAs were significantly associated with prognosis: let-7 g: $\mathrm{HR}=0.74$ (CI: 0.580.93), miR-34a-5p: HR $=0.66(\mathrm{CI}: 0.46-0.94)$, miR-187: $\mathrm{HR}=1.51$ (CI: 1.01-2.24), and miR-205-5p: HR = 0.74(CI: 0.63-0.86). In the adjusted analysis (age, sex, tumor stage/differentiation, ASA-score), low expression of miR34a-5p: HR $=0.58$ (CI: $0.38-0.89$ ) and miR-450b-5p: HR $=0.48(\mathrm{CI}: 0.23-0.99)$ and high expression of miR-187: 
Table 4 Prognostic miRNAs in patients with $P C+A-A C, P C$ and $A-A C$ PDAC and A-AC

CT-expression (per IQR increase)

$\begin{array}{llll} & \text { Unadjusted } & & \\ \text { miRNA } & \text { HR (Cl) } & P & N \\ \text { miR-29a-5p } & 0.87(0.76-0.99) & 0.0302 & 156 \\ \text { miR-34a-5p } & 0.66(0.54-0.81) & <0.0001 & 156 \\ \text { miR-125a-3p } & 0.83(0.73-0.95) & 0.0051 & 153 \\ \text { miR-146a-5p } & 0.87(0.76-0.99) & 0.0296 & 157 \\ \text { miR-205-5p } & 0.91(0.86-0.96) & 4 \mathrm{e}-04 & 130 \\ \text { miR-212-3p } & 0.81(0.72-0.91) & 4 \mathrm{e}-04 & 156\end{array}$

Adjusted

Under median vs. over median

\section{Unadjusted}

miRNA

$\mathrm{HR}(\mathrm{Cl})$

P

N

let-7 $\mathrm{g}$

NS

miR-29a-5p

NS

miR-34a-5p

$0.46(0.31-0.67)$

$<0.0001$

156

miR-205-5p

$0.37(0.25-0.57)$

$<0.0001$

130

5e-04

156

miR-212-3p

$0.51(0.35-0.74)$

0.0495

152

PDAC

CT-expression (per IQR increase)

$\begin{array}{ll} & \text { Unadjusted } \\ \text { miRNA } & H R(C l) \\ \text { miR-34a-5p } & 0.72(0.56-0.93) \\ \text { miR-212-3p } & 0.83(0.71-0.99)\end{array}$

P

0.0104

N

0.0328

103

Under median vs. over median

$\begin{array}{llll} & \text { Unadjusted } & & \\ \text { miRNA } & \text { HR }(C l) & P & N \\ \text { miR-34a-5p } & 0.49(0.31-0.77) & 0.0020 & 103 \\ \text { miR-212-3p } & 0.64(0.41-0.98) & 0.0417 & 103 \\ \text { A-AC } & & & \end{array}$

CT-expression (per IQR increase)

$\begin{array}{lll} & \text { Unadjusted } & \\ \text { miRNA } & H R(C l) & P \\ \text { let-7 g } & 0.74(0.58-0.93) & 0.0100 \\ \text { miR-34a-5p } & 0.66(0.46-0.94) & 0.0218 \\ \text { miR-187 } & 1.51(1.01-2.24) & 0.0439 \\ \text { miR-205-5p } & 0.73(0.63-0.86) & 0.0001 \\ \text { miR-450b-5p } & \text { NS } & \\ \text { Under median vs over } & \end{array}$

\begin{tabular}{llll} 
& Unadjusted & & \\
miRNA & HR $(\mathrm{Cl})$ & $\mathrm{P}$ & $\mathrm{N}$ \\
miR-34a-5p & $0.40(0.19-0.86)$ & 0.0183 & 53 \\
\hline
\end{tabular}

$0.70(0.52-0.93)$
$\mathrm{HR}(\mathrm{Cl})$

$0.85(0.74-0.98)$

$0.64(0.52-0.79)$

$0.83(0.72-0.95)$

$0.85(0.74-0.97)$

$0.92(0.87-0.97)$

$0.80(0.71-0.91)$

$\begin{array}{ll}P & N \\ 0.0212 & 145 \\ <0.0001 & 145 \\ 0.0077 & 142 \\ 0.0191 & 146 \\ 0.0037 & 120 \\ 4 e-04 & 145\end{array}$

Adjusted

$\mathrm{HR}(\mathrm{Cl})$

P

N

$0.62(0.41-0.93)$

0.0220

145

$0.64(0.42-0.96)$

0.0314

145

$0.47(0.31-0.71)$

0.0003

145

$0.44(0.28-0.69)$

0.0003

120

$0.53(0.35-0.79)$

0.0021

145
103

Adjusted

HR (Cl)

$0.82(0.68-0.99)$

Adjusted

HR

$0.53(0.32-0.89)$

$0.59(0.36-0.97)$

(CI)

P

0.0151

0.0358

$P$
0.0144

N

0.0350

93

93

03

$N$
53
53
24
37

Adjusted
Under median vs. over median

$\mathrm{HR}(\mathrm{Cl})$

NS

$0.58(0.38-0.89)$

$2.34(1.22-4.48)$

NS

$0.48(0.23-0.99)$

26

Adjusted

HR (Cl)

$0.36(0.16-0.85)$

P

0.0121

0.0104

52

24

NS Not significant 


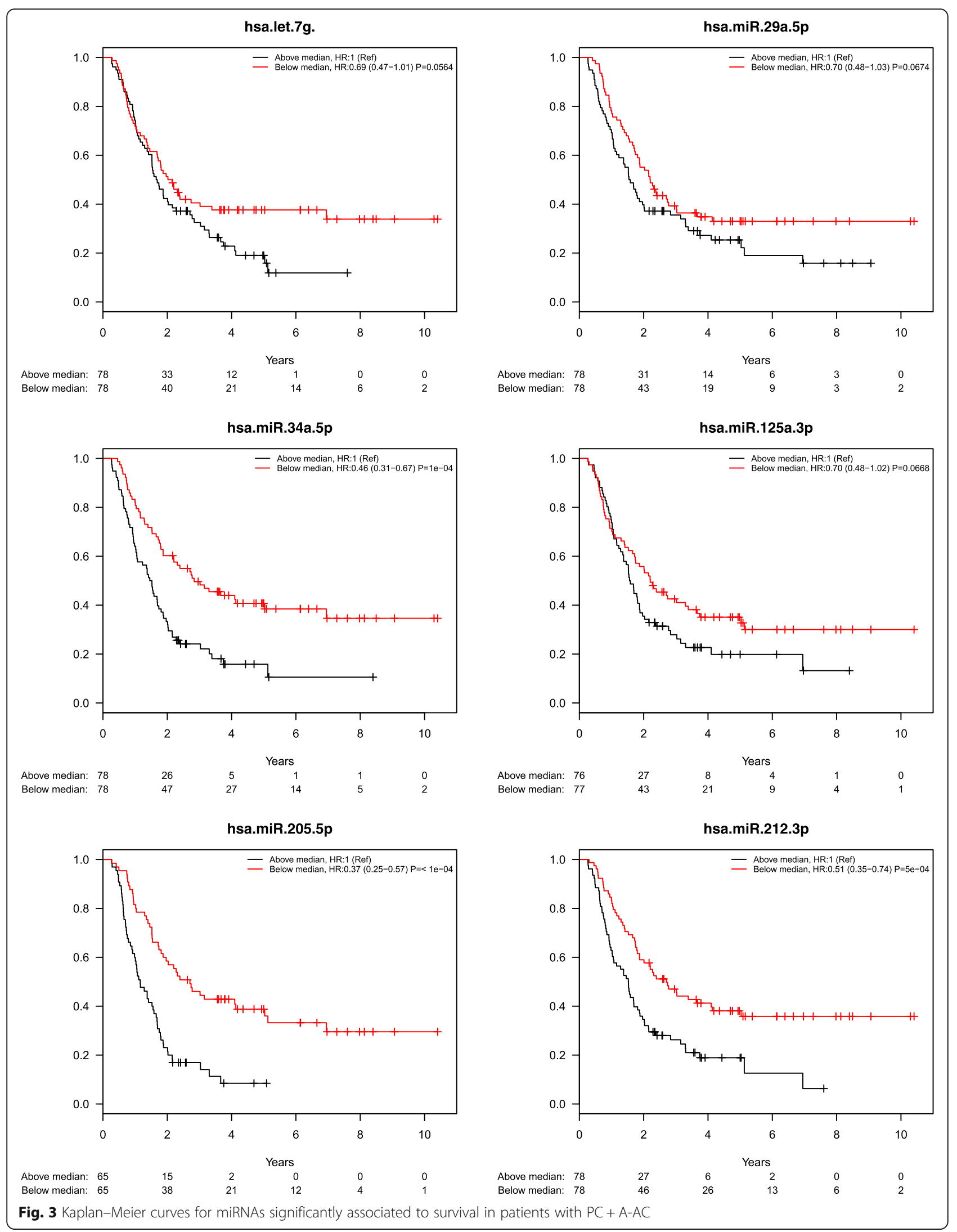


Table 5 Differences of miRNA

\begin{tabular}{|c|c|c|c|c|c|c|c|c|c|}
\hline \multicolumn{5}{|c|}{ Unadjusted effects on differences } & \multicolumn{5}{|c|}{ Adjusted effects on differences } \\
\hline miRNA1 & miRNA2 & $\mathrm{HR}(\mathrm{Cl})$ & $P$ & $\mathrm{~N}$ & miRNA1 & miRNA2 & $\mathrm{HR}(\mathrm{Cl})$ & $P$ & $\mathrm{~N}$ \\
\hline \multicolumn{10}{|l|}{$\mathrm{PDAC}+\mathrm{AAC}$} \\
\hline miR-148a & miR-212-3p & $1.20(1.09-1.33)$ & 0.0002 & 155 & miR-34a-5p & miR-148a & $0.82(0.73-0.92)$ & 0.0011 & 144 \\
\hline miR-205-5p & miR-769-5p & $0.90(0.85-0.95)$ & 0.0003 & 129 & miR-205-5p & miR-769-5p & $0.91(0.85-0.96)$ & 0.0015 & 119 \\
\hline miR-148a & miR-205-5p & $1.08(1.04-1.13)$ & 0.0004 & 130 & miR-146a-5p & miR-212-3p & $1.33(1.11-1.60)$ & 0.0017 & 145 \\
\hline miR-34a-5p & miR-148a & $0.83(0.75-0.92)$ & 0.0009 & 155 & $\operatorname{miR}-34 a-5 p$ & miR-187 & $0.67(0.52-0.88)$ & 0.0038 & 44 \\
\hline miR-34a-5p & miR-187 & $0.64(0.50-0.83)$ & 0.0009 & 47 & miR-148a & miR-205-5p & $1.07(1.02-1.12)$ & 0.004 & 120 \\
\hline miR-146a-5p & miR-212-3p & $1.32(1.12-1.57)$ & 0.0013 & 156 & miR-29a-5p & miR-205-5p & $1.08(1.03-1.15)$ & 0.0046 & 119 \\
\hline miR-187 & miR-212-3p & $1.55(1.18-2.04)$ & 0.0016 & 47 & miR-125a-3p & miR-769-5p & $0.81(0.69-0.94)$ & 0.0071 & 140 \\
\hline miR-34a-5p & miR-769-5p & $0.74(0.62-0.89)$ & 0.0017 & 154 & miR-187 & miR-212-3p & $1.47(1.11-1.96)$ & 0.0078 & 44 \\
\hline miR-212-3p & miR-769-5p & $0.81(0.70-0.92)$ & 0.0020 & 154 & let-7 g & miR-187 & $0.74(0.59-0.93)$ & 0.0085 & 44 \\
\hline miR-205-5p & miR-625-5p & $0.91(0.86-0.97)$ & 0.0023 & 72 & miR-146a-5p & miR-205-5p & $1.08(1.02-1.14)$ & 0.0097 & 120 \\
\hline miR-205-5p & miR-450b-5p & $0.91(0.86-0.97)$ & 0.0031 & 94 & miR-205-5p & miR-222-5p & $0.93(0.87-0.99)$ & 0.0152 & 117 \\
\hline miR-146a-5p & miR-205-5p & $1.08(1.03-1.14)$ & 0.0033 & 130 & miR-29a-5p & miR-769-5p & $0.81(0.68-0.96)$ & 0.0171 & 143 \\
\hline miR-205-5p & miR-222-5p & $0.92(0.86-0.97)$ & 0.0034 & 127 & let-7 g & miR-205-5p & $1.07(1.01-1.13)$ & 0.018 & 120 \\
\hline let-7 g & miR-205-5p & $1.08(1.02-1.14)$ & 0.0048 & 130 & miR-29a-5p & miR-194-3p & $0.68(0.50-0.94)$ & 0.0188 & 46 \\
\hline miR-194-3p & miR-205-5p & $1.26(1.07-1.48)$ & 0.0062 & 36 & miR-125a-3p & miR-187 & $0.76(0.61-0.96)$ & 0.0188 & 43 \\
\hline miR-29a-5p & miR-205-5p & $1.07(1.02-1.13)$ & 0.0072 & 129 & let-7 g & miR-212-3p & $1.14(1.02-1.28)$ & 0.0233 & 144 \\
\hline miR-125a-3p & miR-205-5p & $1.08(1.02-1.15)$ & 0.0074 & 128 & miR-125a-3p & miR-205-5p & $1.07(1.01-1.14)$ & 0.0236 & 118 \\
\hline let-7 g & miR-187 & $0.82(0.70-0.95)$ & 0.0093 & 47 & miR-205-5p & miR-450b-5p & $0.93(0.87-0.99)$ & 0.024 & 85 \\
\hline miR-34a-5p & miR-205-5p & $1.07(1.02-1.13)$ & 0.0125 & 130 & miR-34a-5p & miR-194-3p & $0.64(0.43-0.94)$ & 0.0262 & 45 \\
\hline miR-125a-3p & miR-148a & $0.90(0.83-0.98)$ & 0.0139 & 152 & miR-194-3p & miR-212-3p & $1.39(1.04-1.85)$ & 0.0273 & 45 \\
\hline miR-125a-3p & miR-769-5p & $0.84(0.73-0.97)$ & 0.0146 & 151 & miR-212-3p & miR-625-5p & $0.86(0.75-0.98)$ & 0.0298 & 74 \\
\hline miR-125a-3p & miR-187 & $0.80(0.66-0.96)$ & 0.0155 & 46 & miR-34a-5p & miR-205-5p & $1.07(1.01-1.13)$ & 0.0307 & 120 \\
\hline miR-212-3p & miR-625-5p & $0.87(0.77-0.98)$ & 0.0194 & 79 & miR-194-3p & miR-205-5p & $1.21(1.02-1.45)$ & 0.0326 & 33 \\
\hline let-7 g & miR-212-3p & $1.12(1.01-1.25)$ & 0.0332 & 155 & miR-625-5p & miR-944 & $1.51(1.03-2.22)$ & 0.0339 & 20 \\
\hline miR-187 & miR-194-3p & $1.41(1.02-1.96)$ & 0.0366 & 21 & miR-125a-3p & miR-148a & $0.91(0.84-1.00)$ & 0.0383 & 141 \\
\hline miR-205-5p & miR-212-3p & $0.95(0.90-1.00)$ & 0.0410 & 130 & miR-146a-5p & miR-769-5p & $0.84(0.71-1.00)$ & 0.0394 & 144 \\
\hline miR-34a-5p & miR-625-5p & $0.88(0.78-1.00)$ & 0.0443 & 79 & miR-34a-5p & miR-625-5p & $0.87(0.75-1.00)$ & 0.0478 & 74 \\
\hline miR-146a-5p & miR-187 & $0.79(0.63-1.00)$ & 0.0452 & 47 & & & & & \\
\hline miR-187 & miR-205-5p & $1.12(1.00-1.26)$ & 0.0468 & 38 & & & & & \\
\hline miR-34a-5p & miR-146a-5p & $0.83(0.68-1.00)$ & 0.0488 & 156 & & & & & \\
\hline \multicolumn{10}{|l|}{ PDAC } \\
\hline miR-148a & miR-212-3p & $1.18(1.04-1.33)$ & 0.0077 & 103 & miR-34a-5p & miR-769-5p & $0.63(0.47-0.84)$ & 0.002 & 92 \\
\hline miR-34a-5p & miR-148a & $0.86(0.76-0.97)$ & 0.0156 & 103 & miR-29a-5p & miR-187 & $1.99(1.20-3.29)$ & 0.0072 & 20 \\
\hline miR-34a-5p & miR-769-5p & $0.75(0.59-0.96)$ & 0.0199 & 102 & miR-187 & miR-769-5p & $0.54(0.33-0.87)$ & 0.0111 & 20 \\
\hline miR-146a-5p & miR-212-3p & $1.26(1.01-1.56)$ & 0.0371 & 103 & miR-187 & miR-205-5p & $0.72(0.56-0.94)$ & 0.0138 & 19 \\
\hline \multirow[t]{8}{*}{ miR-34a-5p } & miR-146a-5p & $0.74(0.56-0.99)$ & 0.0427 & 103 & $\operatorname{miR}-212-3 p$ & miR-769-5p & $0.75(0.60-0.95)$ & 0.0153 & 92 \\
\hline & & & & & miR-148a & miR-212-3p & $1.18(1.03-1.34)$ & 0.016 & 93 \\
\hline & & & & & miR-450b-5p & miR-944 & $1.56(1.06-2.30)$ & 0.0243 & 24 \\
\hline & & & & & miR-34a-5p & miR-148a & $0.86(0.75-0.99)$ & 0.0341 & 93 \\
\hline & & & & & miR-146a-5p & miR-212-3p & $1.29(1.02-1.63)$ & 0.0343 & 93 \\
\hline & & & & & miR-148a & miR-431-5p & $1.32(1.02-1.72)$ & 0.0364 & 34 \\
\hline & & & & & miR-146a-5p & miR-187 & $1.57(1.01-2.44)$ & 0.0438 & 20 \\
\hline & & & & & miR-222-5p & miR-769-5p & $0.84(0.70-1.00)$ & 0.0491 & 92 \\
\hline
\end{tabular}


Table 5 Differences of miRNA (Continued)

\begin{tabular}{|c|c|c|c|c|c|c|c|c|c|}
\hline \multicolumn{10}{|l|}{$A-A C$} \\
\hline miR-205-5p & miR-769-5p & $0.71(0.60-0.84)$ & $<0.0001$ & 36 & miR-34a-5p & miR-769-5p & $0.51(0.32-0.81)$ & 0.0043 & 51 \\
\hline $\operatorname{miR}-34 a-5 p$ & miR-187 & $0.44(0.27-0.72)$ & 0.0011 & 24 & miR-125a-3p & miR-187 & $0.37(0.18-0.75)$ & 0.0055 & 23 \\
\hline miR-148a & miR-205-5p & $1.25(1.09-1.44)$ & 0.0018 & 37 & miR-34a-5p & miR-187 & $0.48(0.28-0.82)$ & 0.0067 & 24 \\
\hline miR-125a-3p & miR-187 & $0.69(0.54-0.88)$ & 0.0032 & 23 & miR-148a & miR-187 & $0.59(0.40-0.87)$ & 0.0074 & 24 \\
\hline miR-187 & miR-205-5p & $1.35(1.10-1.66)$ & 0.0041 & 17 & miR-29a-5p & miR-769-5p & $0.65(0.48-0.89)$ & 0.0077 & 52 \\
\hline miR-187 & miR-212-3p & $2.22(1.29-3.82)$ & 0.0042 & 24 & miR-222-5p & miR-450b-5p & $2.12(1.18-3.81)$ & 0.0123 & 25 \\
\hline miR-205-5p & miR-450b-5p & $0.73(0.59-0.91)$ & 0.0045 & 22 & miR-187 & miR-769-5p & $2.09(1.16-3.78)$ & 0.0148 & 24 \\
\hline let-7 g & miR-205-5p & $1.28(1.07-1.52)$ & 0.006 & 37 & miR-29a-5p & miR-187 & $0.62(0.42-0.91)$ & 0.0154 & 24 \\
\hline miR-146a-5p & miR-205-5p & $1.19(1.05-1.34)$ & 0.0065 & 37 & miR-187 & miR-212-3p & $2.23(1.16-4.30)$ & 0.016 & 24 \\
\hline let-7 g & miR-769-5p & $0.74(0.59-0.93)$ & 0.0083 & 52 & miR-146a-5p & miR-187 & $0.54(0.33-0.90)$ & 0.0175 & 24 \\
\hline miR-34a-5p & miR-769-5p & $0.66(0.48-0.91)$ & 0.0122 & 52 & miR-148a & miR-450b-5p & $2.12(1.14-3.96)$ & 0.0181 & 26 \\
\hline miR-34a-5p & miR-205-5p & $1.22(1.04-1.43)$ & 0.0126 & 37 & miR-450b-5p & miR-769-5p & $0.31(0.12-0.84)$ & 0.0214 & 26 \\
\hline let-7 g & miR-187 & $0.77(0.62-0.95)$ & 0.017 & 24 & miR-34a-5p & miR-625-5p & $0.71(0.52-0.96)$ & 0.0267 & 30 \\
\hline let-7 g & miR-625-5p & $0.74(0.58-0.95)$ & 0.0175 & 31 & miR-125a-3p & miR-769-5p & $0.75(0.58-0.97)$ & 0.0283 & 49 \\
\hline miR-125a-3p & miR-205-5p & $1.21(1.03-1.43)$ & 0.0227 & 36 & miR-29a-5p & miR-625-5p & $0.74(0.56-0.99)$ & 0.0408 & 30 \\
\hline let-7 g & miR-222-5p & $0.80(0.67-0.97)$ & 0.0242 & 50 & miR-205-5p & miR-222-5p & $0.81(0.66-0.99)$ & 0.0436 & 33 \\
\hline $\operatorname{miR}-29 a-5 p$ & miR-187 & $0.74(0.56-0.97)$ & 0.0272 & 24 & & & & & \\
\hline miR-205-5p & miR-212-3p & $0.86(0.75-0.98)$ & 0.0289 & 37 & & & & & \\
\hline miR-146a-5p & miR-187 & $0.67(0.46-0.96)$ & 0.0308 & 24 & & & & & \\
\hline miR-187 & miR-769-5p & $1.47(1.02-2.11)$ & 0.0367 & 24 & & & & & \\
\hline miR-450b-5p & miR-769-5p & $0.59(0.35-1.00)$ & 0.0489 & 27 & & & & & \\
\hline
\end{tabular}

$\mathrm{HR}=2.34(\mathrm{CI}: 1.22-4.48)$ were associated with short OS. When patients were divided into 2 groups for each miRNA (defined as expression under or above the median level), low expression of miR-34a-5p was associated with short OS. Figure 4 shows Kaplan-Meier curves for the miRNAs reaching a significance level below 0.01 .

Table 5 shows 21 and 16 combinations of 2 miRNAs in A-AC FFPE tissue significantly associated with short OS in both an unadjusted and an adjusted analysis.

\section{Discussion}

In the present study, our aim was to validate previously described tissue miRNA expression profiles as diagnostic and prognostic biomarkers of $\mathrm{PC}$ and other periampullary cancers [20-32]. We used non-microdissected FFPE tissue from 165 patients who had undergone surgery for PDAC and from 86 patients who had undergone resection for other periampullary cancers.

Many of the diagnostic miRNAs described in the literature $[20,21,34]$ could be validated. We found the following miRNAs either upregulated or downregulated in PC tissue compared to tissue from CP and/or normal pancreas, upregulated miRNAs: miR-21-5p, -23a-3p, $-31-5 \mathrm{p},-34 \mathrm{c}-5 \mathrm{p},-93-3 \mathrm{p},-135 \mathrm{~b}-3 \mathrm{p},-155-5 \mathrm{p},-186-5 \mathrm{p}$, $-196 \mathrm{~b}-5 \mathrm{p},-203,-205-5 \mathrm{p},-210,-222-3 \mathrm{p},-451,-492$, -614, and miR-622; and downregulated miRNAs: miR- 122-5p, -130b-3p, -216b, -217, and miR-375. Furthermore, we validated the two-miRNA index "miR-196b miR-217" [27], and suggested new diagnostic indices for separating patients with PC vs. HS and PC vs. HS and $\mathrm{CP}$ combined. We found that these indices were useful in discriminating other upper gastrointestinal cancers (duodenal cancer, common bile duct cancer and gastric cancer) from normal pancreas and CP.

In addition to the diagnostic miRNAs, we demonstrated the association of 10 miRNAs with prognosis and constructed several indices based on differences of 2 miRNA associated with poor prognosis.

A major limitation of the study was the high number of non-detectable miRNAs using the Fluidigm BioMark System ${ }^{\mathrm{Ts}}$. Even though we purified the miRNAs from FFPE by the same method as in our previous studies [27, 31] and repeated the analysis several times, we still experienced a high number of undetectable miRNAs. At present, we have no explanation for this problem apart from possible platform sensitivity limitations.

We consider it a strength of the study that nonmicrodissected samples were used, since this will also be the case in a clinical setting. The tumor microenvironment is a highly dynamic component of PC, often constitutes the bulk of the tumor, and should therefore be 


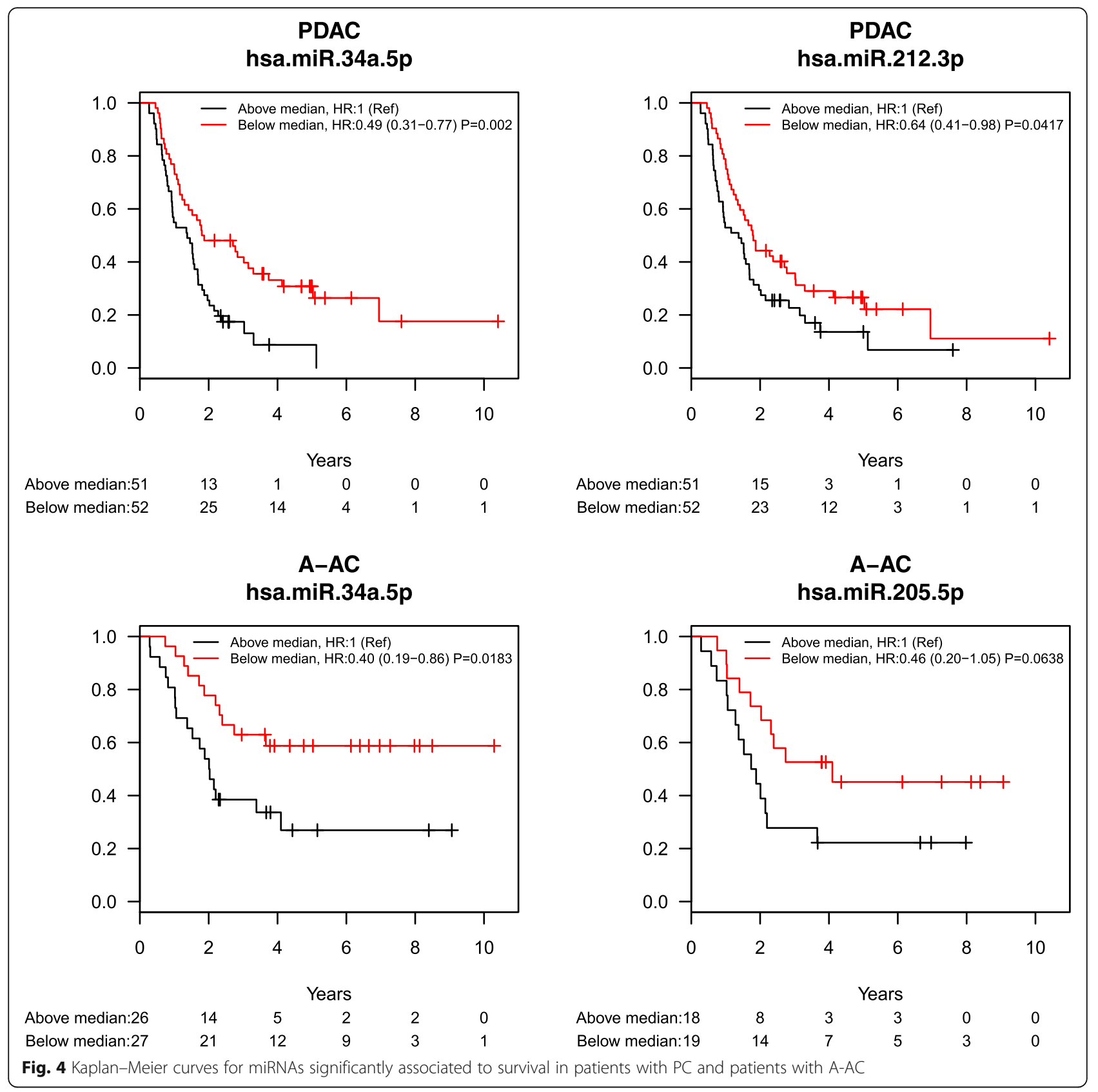

taken into account. The extracellular stroma participates in paracrine signaling that promotes PDAC cell survival and metastasis, and the dense extracellular matrix characteristic of PDAC acts as a physical barrier to infiltrating immune cells and the diffusion of chemotherapy [35-37]. MicroRNAs are involved in the regulation of the extracellular components in different tissues [38, 39]. Since many studies regarding miRNAs in PC are performed on microdissected tissue or cell lines the miRNAs originating from the extracellular stroma are less elucidated. The following miRNAs significantly deregulated in the present study are known to be related to the extracellular compartment of PC: miR-21, $-29,-130 \mathrm{~b}$, -210 , and -451 [40-43].

Among the validated miRNAs, high expression of miR-21, miR-31, and miR-155 and low expression of miR-217 and miR-375are the most consistently described dysregulated miRNAs in PC. Several studies have found miR-155to be upregulated in PC [20-22, 28, 32, 44, 45]. miR-155 functions as an onco-miRNA in different types of cancer,e.g., breast, cervix, colon, and lung cancer, and high miR-155 expression in cancer tissue is associated with poor prognosis in PC and lung cancer [30, 46-49]. The oncogenic effect of miR-155 maybe 
caused by the targeting of anti-inflammatory signal pathways such as Sh2 domain-containing inositol phosphatase-1 (Ship1) or from suppression of cytokine signaling 1 (Socs1) [50, 51].

miR-21 is also an onco-miR involved in PC tumorigenesis, invasion, metastasis, and chemoresistance [20, 21, $23,27,32,44,45,52-57]$. miR-21 is primarily upregulated in the extracellular stroma, which is considered a dynamic component of PC, and high expression is associated with poor prognosis [40]. Our study was conducted on non-microdissected tissue and thus also detects miRNAs in the extracellular stroma.miR-21 targets tumor suppressors like PTEN, PDCD4, and TIMP3, components of the p53 pathway, and modulates TGF-b signaling, thus promoting cell proliferation, survival, and migration/invasion [45, 58-60].

miR-31 is upregulated in PC $[21,27,28,45,61]$. miR31 targets human mutL homolog 1 (a mismatch repair protein) [62] and activates the RAS pathway by inhibiting RAS p21 GTPase activating protein 1 (RASA1) in colorectal cancer [63].

miR-217 is downregulated in $\mathrm{PC}$ and in pancreatic intraepithelial neoplasm (PanIN) [21, 27, 28, 32, 45, 64]. This finding has also been replicated in studies using fine needle aspirates from PC $[24,65] . \mathrm{miR}-217$ acts as a tumor suppressor in PC by targeting KRAS [66] and is involved in epithelial-mesenchymal-transition (EMT) in PC and CP via the miR-217-SIRT1 pathway, which can be triggered by TGF- $\beta 1$ in inflammatory processes [67].

miR-375 is downregulated in PC compared to normal pancreas, is associated with prognosis, and can differentiate between pancreatobiliary and intestinal subtypes in ampullary adenocarcinoma $[20,21,27,28$, 32, 68]. miR-375 is also downregulated in esophageal, gastric, breast, lung, colorectal, and cervical cancers [69-74]. miR-375 plays a role in the development and maintenance of the $\alpha$ - and $\beta$-cell mass in the normal pancreas and is upregulated in patients with type 2 diabetes [75, 76].miR-375 targets 3-phosphoinositidedependent protein kinase-1 (PDK1) in PC and inhibits PC cell proliferation in vitro $[77,78]$.

In the literature, the following miRNAs are described as prognostic after PC resection:Let-7 g, miR-21, miR29a-5p, miR-34a-5p, miR-146a, miR-155, miR-196a, miR-203, miR-205, miR-210, miR-212, miR-222, miR450b-5p, and miR-675 [23, 29-32]. We have previously described prognostic indices using combinations of high expression of miR-212 and miR-675 and low expression of miR-148a-5p (previous ID: miR-148a*), miR-187 and let-7 g-3p (previous ID: let-7 $\mathrm{g}^{*}$ ) in FFPE tissue from patients operated for PC [31]. Only a few of these patients received adjuvant chemotherapy after surgery. In the present study, patients with PDAC and A-AC were all treated with adjuvant gemcitabine for 6 months or until disease recurrence. In this population, we could validate let-7 g, miR-29a-5p, miR-34a-5p, miR-146a-5p, miR-205-5p, and miR-212-3pas prognostic biomarkers after radical resection for PC.

The let-7 family of miRNAs includes tumor suppressor miRNAs, the expression of which is prognostic in HCC, gastric, and ovarian cancers [79-81]. Let-7 $\mathrm{g}$ is involved in pathways essential for the development of cancer. It targets Fas and is involved in Fas-mediated apoptosis [82]. Silencing of let-7b/g activates AKT signaling and promotes carcinogenesis in gastric cancer [83]. Let-7 inhibits cell motility in breast cancer by regulating genes in the cytoskeleton pathway and silencing of let-7 promotes metastases [84]. Let-7 inhibits proliferation in $\mathrm{HCC}$ by downregulation of c-Myc and upregulation of p16(INK4A) [85].

In PC, miR-29a-5p induces EMT, stimulates pancreatic stellate cells to accumulate protein in the extracellular matrix, and increases resistance to gemcitabine through the Wnt/beta-catenin pathway [41, 86, 87]. miR-34a is upregulated in cervical and colorectal cancers and downregulated in breast, prostate, renal and lung cancer [49, 88].

The miR-34 family miRNAs are described as tumor suppressor miRNAs, and miR-34a/c suppresses breast cancer invasion and metastasis by targeting Fos-related antigen-1 [89]. PC mouse models show that miR-146a acts through EGFR signaling [90]. miR-205 is involved in EMT and acts through the anti-apoptotic protein Bcl-2 (in prostate cancer) and HER3 (in breast cancer) [91-93]. We found that low expression of miR$125 a-3 p$ was associated with short OS in patients with $\mathrm{PC}$, and this is a novel observation.miR-125a-3p has been described as a tumor suppressor miRNA in several cancers $[94,95]$.

In the present study, miR-130b was found to be downregulated in PDAC compared to benign specimens. Interestingly, this miRNA is upregulated in the stroma compared to carcinoma cells [42].

Further information about the 46 miRNAs analyzed in the present study is given in "Additional file 1".

\section{Conclusions}

In conclusion, we could validate miRNAs selected from the literature as diagnostic and/or prognostic biomarkers in patients radically resected for PC. No microdissection of the tumors was done, and some of the miRNAs most likely originated from the stroma and not the cancer cells. The diagnostic ability of these miRNAs was also tested on duodenal cancer, common bile duct cancer, and gastric cancer - diagnoses that represent a considerable diagnostic challenge in separating from $\mathrm{PC}$ in a clinical setting. Hopefully, this study can contribute to the understanding of pancreatic and periampullary 
cancers and improve the diagnosis, prognosis, and ultimately treatment of patients with these conditions. For example, this could be achieved by allocating young patients with a miRNA expression profile suggestive of poor prognosis to a more aggressive chemotherapy regimen, or elderly patients with a more promising prognostic profile could be spared from adjuvant therapy.

\section{Additional files}

Additional file 1: Background on all measured microRNA. (DOCX 1012 kb)

Additional file 2: All statistical calculations including insignificant results not presented in the manuscript. (DOC $1481 \mathrm{~kb}$ )

\section{Abbreviations}

A-AC: Ampullary adenocarcinoma; CBD: Common bile duct; CP: Chronic pancreatitis; DC: Duodenal cancer; FFPE: Formalin-fixed paraffin-embedded; GC: Gastric cancer; HS: Healthy subjects; miR: microRNA; miRNA: microRNA; PC: Pancreatic cancer; PDAC: Pancreatic ductal adenocarcinoma

\section{Acknowledgements}

We thank Dr. Nathalia A. Giese, MD, PhD, Heidelberg, Germany, for providing tissue samples for this study.

\section{Funding}

Professor Molven received a grant from Western Norway Regional Health Authority (Helse Vest).

\section{Availability of data and materials}

The datasets used and/or analyzed during the current study available from the corresponding author on reasonable request. All calculations are included in the manuscript or submitted in "Additional file 2".

\section{Authors' contributions}

DC designed the study, collected the specimens from Denmark and the corresponding clinical data, interpreted the calculations wrote the manuscript; CD performed all calculations and contributed to the manuscript; MKB contributed to the manuscript; JPH re-assessed all the specimens from Denmark; NAS contributed to data interpretation and to the manuscript; JW contributed with the German specimens; $\mathrm{HI}$ contributed with the Norwegian specimens; AM contributed with the Norwegian specimens and contributed to the manuscript; $\mathrm{CPH}$ contributed with clinical data and contributed to the manuscript. JSJ designed the study, contributed with collection of specimens, clinical data, interpretation of the calculations, preparation of the manuscript and funding. All authors read and approved the final manuscript.

\section{Competing interests}

The authors declare that they have no competing interests.

\section{Consent for publication}

Not applicable.

\section{Ethics approval and consent to participate}

The patients included in the BIOPAC Study provided written informed consent. The study was approved by the Regional Ethics Committee (VEK ref. KA-20060113) and the Danish Data Protection Agency (j.nr. 2006-41-6848, jr. nr. 2012-58-004, and HGH-2015-027, I-suite 03960). The collection of archived FFPE tissues from CBD cancer and GC was approved by the local ethics committee. The samples from Heidelberg and Bergen were obtained from patients included in studies approved by their local ethics committees.

\section{Author details}

'Department of Surgical Gastroenterology and Transplantation, Rigshospitalet, Copenhagen University Hospital, Copenhagen, Denmark.
${ }^{2}$ Danish Cancer Society Research Center, Danish Cancer Society, Copenhagen, Denmark. ${ }^{3}$ Department of Oncology, Herlev and Gentofte Hospital, Copenhagen University Hospital, Herlev, Denmark. ${ }^{4}$ Department of Pathology, Rigshospitalet, Copenhagen University Hospital, Copenhagen, Denmark. ${ }^{5}$ Department of General, Visceral, and Transplant Surgery, LMU, University of Munich, Munich, Germany. ${ }^{6}$ Gade Laboratory for Pathology, Department of Clinical Medicine, University of Bergen, Bergen, Norway. ${ }^{7}$ Department of Pathology, Ålesund Hospital, Ålesund, Norway. ${ }^{8}$ Department of Pathology, Haukeland University Hospital, Bergen, Norway. ${ }^{9}$ Department of Medicine, Herlev and Gentofte Hospital, Copenhagen University Hospital, Herlev, Denmark. ${ }^{10}$ Institute of Clinical Medicine, Faculty of Health and Medical Sciences, University of Copenhagen, Copenhagen, Denmark. ${ }^{11}$ Department of Oncology, Herlev University Hospital, Herlev Ringvej 75, DK-2730 Herlev, Denmark.

Received: 28 October 2016 Accepted: 3 February 2017

Published online: 21 February 2017

\section{References}

1. Ryan DP, Hong TS, Bardeesy N. Pancreatic adenocarcinoma. N Engl J Med. 2014;371(11):1039-49.

2. Siegel RL, Miller KD, Jemal A. Cancer statistics, 2016. CA Cancer J Clin. 2016: 66(1):7-30.

3. Yeo CJ, Cameron JL, Lillemoe KD, Sitzmann JV, Hruban RH, Goodman SN, Dooley WC, Coleman J, Pitt HA. Pancreaticoduodenectomy for cancer of the head of the pancreas. 201 patients. Ann Surg. 1995;221(6):721-31. discussion 731-723.

4. Cancer Research UK. Cancer mortality: UK Statistics. In.; 2009. http://www. cancerresearchuk.org/health-professional/cancer-statistics/statistics-bycancer-type/pancreatic-cancer/mortality. (Accessed 15th Mar 2016).

5. Hartwig W, Hackert T, Hinz U, Gluth A, Bergmann F, Strobel O, Buchler MW, Werner J. Pancreatic cancer surgery in the new millennium: better prediction of outcome. Ann Surg. 2011;254(2):311-9.

6. He J, Ahuja N, Makary MA, Cameron JL, Eckhauser FE, Choti MA, Hruban RH, Pawlik TM, Wolfgang CL. 2564 resected periampullary adenocarcinomas at a single institution: trends over three decades. HPB (Oxford). 2014;16(1):83-90.

7. Bettschart V, Rahman MQ, Engelken FJ, Madhavan KK, Parks RW, Garden OJ. Presentation, treatment and outcome in patients with ampullary tumours. Br J Surg. 2004;91(12):1600-7.

8. Struck A, Howard T, Chiorean EG, Clarke JM, Riffenburgh R, Cardenes HR. Non-ampullary duodenal adenocarcinoma: factors important for relapse and survival. J Surg Oncol. 2009;100(2):144-8.

9. Seufferlein T, Bachet JB, Van Cutsem E, Rougier P. Pancreatic adenocarcinoma: ESMO-ESDO Clinical Practice Guidelines for diagnosis, treatment and follow-up. Ann Oncol. 2012;23 Suppl 7:vii33-40.

10. Waldman SA, Terzic A. Translating MicroRNA discovery into clinical biomarkers in cancer. JAMA. 2007;297(17):1923-5.

11. Nelson KM, Weiss GJ. MicroRNAs and cancer: past, present, and potential future. Mol Cancer Ther. 2008;7(12):3655-60.

12. Ambros $V$. The functions of animal microRNAs. Nature. 2004:431(7006):350-5.

13. Hwang HW, Mendell JT. MicroRNAs in cell proliferation, cell death, and tumorigenesis. Br J Cancer. 2006;94(6):776-80.

14. Li M, Marin-Muller C, Bharadwaj U, Chow KH, Yao Q, Chen C. MicroRNAs: control and loss of control in human physiology and disease. World J Surg. 2009;33(4):667-84.

15. Bartel DP. MicroRNAs: target recognition and regulatory functions. Cell. 2009;136(2):215-33.

16. Almeida MI, Reis RM, Calin GA. MicroRNA history: discovery, recent applications, and next frontiers. Mutat Res. 2011;717(1-2):1-8.

17. Li J, Smyth P, Flavin R, Cahill S, Denning K, Aherne S, Guenther SM, O'Leary $\mathrm{J}$, Sheils O. Comparison of miRNA expression patterns using total RNA extracted from matched samples of formalin-fixed paraffin-embedded (FFPE) cells and snap frozen cells. BMC Biotechnol. 2007;7:36.

18. Zhang X, Chen J, Radcliffe T, Lebrun DP, Tron VA, Feilotter H. An arraybased analysis of microRNA expression comparing matched frozen and formalin-fixed paraffin-embedded human tissue samples. J Mol Diagn. 2008;10(6):513-9.

19. MirBase [http://www.mirbase.org]. Accessed 15th Mar 2016.

20. Bloomston M, Frankel WL, Petrocca F, Volinia S, Alder H, Hagan JP, Liu CG, Bhatt D, Taccioli C, Croce CM. MicroRNA expression patterns to differentiate 
pancreatic adenocarcinoma from normal pancreas and chronic pancreatitis. JAMA. 2007;297(17):1901-8.

21. Szafranska AE, Davison TS, John J, Cannon T, Sipos B, Maghnouj A, Labourier E, Hahn SA. MicroRNA expression alterations are linked to tumorigenesis and non-neoplastic processes in pancreatic ductal adenocarcinoma. Oncogene. 2007;26(30):4442-52.

22. Lee EJ, Gusev Y, Jiang J, Nuovo GJ, Lerner MR, Frankel WL, Morgan DL, Postier RG, Brackett DJ, Schmittgen TD. Expression profiling identifies microRNA signature in pancreatic cancer. Int J Cancer. 2007;120(5):1046-54.

23. Dillhoff M, Liu J, Frankel W, Croce C, Bloomston M. MicroRNA-21 is overexpressed in pancreatic cancer and a potential predictor of survival. J Gastrointest Surg. 2008;12(12):2171-6.

24. Szafranska AE, Doleshal M, Edmunds HS, Gordon S, Luttges J, Munding JB, Barth Jr RJ, Gutmann EJ, Suriawinata AA, Marc Pipas J, et al. Analysis of microRNAs in pancreatic fine-needle aspirates can classify benign and malignant tissues. Clin Chem. 2008;54(10):1716-24.

25. Habbe N, Koorstra JB, Mendell JT, Offerhaus GJ, Ryu JK, Feldmann G, Mullendore ME, Goggins MG, Hong SM, Maitra A. MicroRNA miR-155 is a biomarker of early pancreatic neoplasia. Cancer Biol Ther. 2009;8(4):340-6.

26. Zhang Y, Li M, Wang H, Fisher WE, Lin PH, Yao Q, Chen C. Profiling of 95 microRNAs in pancreatic cancer cell lines and surgical specimens by realtime PCR analysis. World J Surg. 2009;33(4):698-709.

27. Schultz NA, Werner J, Willenbrock H, Roslind A, Giese N, Horn T, Wojdemann M, Johansen JS. MicroRNA expression profiles associated with pancreatic adenocarcinoma and ampullary adenocarcinoma. Mod Pathol. 2012;25(12):1609-22

28. Bauer AS, Keller A, Costello E, Greenhalf W, Bier M, Borries A, Beier M, Neoptolemos J, Buchler M, Werner J, et al. Diagnosis of pancreatic ductal adenocarcinoma and chronic pancreatitis by measurement of microRNA abundance in blood and tissue. PLoS One. 2012;7(4), e34151.

29. Ikenaga N, Ohuchida K, Mizumoto K, Yu J, Kayashima T, Sakai H, Fujita H, Nakata K, Tanaka M. MicroRNA-203 expression as a new prognostic marker of pancreatic adenocarcinoma. Ann Surg Oncol. 2010;17(12):3120-8.

30. Greither T, Grochola LF, Udelnow A, Lautenschlager C, Wurl P, Taubert H. Elevated expression of microRNAs 155, 203, 210 and 222 in pancreatic tumors is associated with poorer survival. Int J Cancer. 2010;126(1):73-80.

31. Schultz NA, Andersen KK, Roslind A, Willenbrock H, Wojdemann M, Johansen JS. Prognostic microRNAs in cancer tissue from patients operated for pancreatic cancer-five microRNAs in a prognostic index. World J Surg. 2012;36(11):2699-707.

32. Jamieson NB, Morran DC, Morton JP, Ali A, Dickson EJ, Carter CR, Sansom OJ, Evans TR, McKay CJ, Oien KA. MicroRNA molecular profiles associated with diagnosis, clinicopathologic criteria, and overall survival in patients with resectable pancreatic ductal adenocarcinoma. Clin Cancer Res. 2012; 18(2):534-45.

33. Aaltonen LA, Hamilton SR, World Health Organization, International Agency for Research on Cancer. Pathology and genetics of tumours of the digestive system. Lyon, Oxford: IARC Press; Oxford University Press (distributor); 2000.

34. Frampton AE, Giovannetti E, Jamieson NB, Krell J, Gall TM, Stebbing J, Jiao LR, Castellano L. A microRNA meta-signature for pancreatic ductal adenocarcinoma. Expert Rev Mol Diagn. 2014;14(3):267-71.

35. Neesse A, Algul H, Tuveson DA, Gress TM. Stromal biology and therapy in pancreatic cancer: a changing paradigm. Gut. 2015;64(9):1476-84.

36. Hwang RF, Moore T, Arumugam T, Ramachandran V, Amos KD, Rivera A, Ji B, Evans DB, Logsdon CD. Cancer-associated stromal fibroblasts promote pancreatic tumor progression. Cancer Res. 2008;68(3):918-26.

37. Xu Z, Vonlaufen A, Phillips PA, Fiala-Beer E, Zhang X, Yang L, Biankin AV, Goldstein D, Pirola RC, Wilson JS, et al. Role of pancreatic stellate cells in pancreatic cancer metastasis. Am J Pathol. 2010;177(5):2585-96.

38. Chou J, Shahi P, Werb Z. microRNA-mediated regulation of the tumor microenvironment. Cell Cycle. 2013;12(20):3262-71.

39. Zhang $Y$, Yang $P$, Wang XF. Microenvironmental regulation of cancer metastasis by miRNAs. Trends Cell Biol. 2014;24(3):153-60.

40. Kadera BE, Li L, Toste PA, Wu N, Adams C, Dawson DW, Donahue TR. MicroRNA-21 in pancreatic ductal adenocarcinoma tumor-associated fibroblasts promotes metastasis. PLoS One. 2013;8(8), e71978.

41. Kwon JJ, Nabinger SC, Vega Z, Sahu SS, Alluri RK, Abdul-Sater Z, Yu Z, Gore J, Nalepa G, Saxena R, et al. Pathophysiological role of microRNA-29 in pancreatic cancer stroma. Sci Rep. 2015;5:11450.
42. Sandhu V, Bowitz Lothe IM, Labori KJ, Skrede ML, Hamfjord J, Dalsgaard AM, Buanes T, Dube G, Kale MM, Sawant S, et al. Differential expression of miRNAs in pancreatobiliary type of periampullary adenocarcinoma and its associated stroma. Mol Oncol. 2016:10(2):303-16.

43. Takikawa T, Masamune A, Hamada S, Nakano E, Yoshida N, Shimosegawa T. miR-210 regulates the interaction between pancreatic cancer cells and stellate cells. Biochem Biophys Res Commun. 2013;437(3):433-9.

44. Panarelli NC, Chen YT, Zhou XK, Kitabayashi N, Yantiss RK. MicroRNA expression aids the preoperative diagnosis of pancreatic ductal adenocarcinoma. Pancreas. 2012;41(5):685-90.

45. Nagao Y, Hisaoka M, Matsuyama A, Kanemitsu S, Hamada T, Fukuyama T, Nakano R, Uchiyama A, Kawamoto M, Yamaguchi K, et al. Association of microRNA-21 expression with its targets, PDCD4 and TIMP3, in pancreatic ductal adenocarcinoma. Mod Pathol. 2012;25(1):112-21.

46. Volinia S, Calin GA, Liu CG, Ambs S, Cimmino A, Petrocca F, Visone R, lorio M, Roldo C, Ferracin M, et al. A microRNA expression signature of human solid tumors defines cancer gene targets. Proc Natl Acad Sci U S A. 2006;103(7):2257-61

47. Yanaihara N, Caplen N, Bowman E, Seike M, Kumamoto K, Yi M, Stephens RM, Okamoto A, Yokota J, Tanaka T, et al. Unique microRNA molecular profiles in lung cancer diagnosis and prognosis. Cancer Cell. 2006;9(3):189-98.

48. Saito M, Schetter AJ, Mollerup S, Kohno T, Skaug V, Bowman ED, Mathe EA, Takenoshita S, Yokota J, Haugen A, et al. The association of microRNA expression with prognosis and progression in early-stage, non-small cell lung adenocarcinoma: a retrospective analysis of three cohorts. Clin Cancer Res. 2011;17(7):1875-82

49. Gocze K, Gombos K, Juhasz K, Kovacs K, Kajtar B, Benczik M, Gocze P, Patczai B, Arany I, Ember I. Unique MicroRNA expression profiles in cervical cancer. Anticancer Res. 2013;33(6):2561-7.

50. Jiang S, Zhang HW, Lu MH, He XH, Li Y, Gu H, Liu MF, Wang ED. MicroRNA155 functions as an OncomiR in breast cancer by targeting the suppressor of cytokine signaling 1 gene. Cancer Res. 2010;70(8):3119-27.

51. Ji Y, Wrzesinski C, Yu Z, Hu J, Gautam S, Hawk NV, Telford WG, Palmer DC, Franco Z, Sukumar M, et al. miR-155 augments CD8+ T-cell antitumor activity in lymphoreplete hosts by enhancing responsiveness to homeostatic gammac cytokines. Proc Natl Acad Sci U S A. 2015;112(2):476-81.

52. Giovannetti E, Funel N, Peters GJ, Del Chiaro M, Erozenci LA, Vasile E, Leon LG, Pollina LE, Groen A, Falcone A, et al. MicroRNA-21 in pancreatic cancer: correlation with clinical outcome and pharmacologic aspects underlying its role in the modulation of gemcitabine activity. Cancer Res. 2010;70(11): 4528-38.

53. Frampton $A E$, Castellano L, Colombo T, Giovannetti E, Krell J, Jacob J, Pellegrino L, Roca-Alonso L, Funel N, Gall TM, et al. MicroRNAs cooperatively inhibit a network of tumor suppressor genes to promote pancreatic tumor growth and progression. Gastroenterology. 2014; 146(1):268-77. e218.

54. Hwang JH, Voortman J, Giovannetti E, Steinberg SM, Leon LG, Kim YT, Funel N, Park JK, Kim MA, Kang GH, et al. Identification of microRNA-21 as a biomarker for chemoresistance and clinical outcome following adjuvant therapy in resectable pancreatic cancer. PLoS One. 2010;5(5):e10630.

55. Moriyama T, Ohuchida K, Mizumoto K, Yu J, Sato N, Nabae T, Takahata S, Toma H, Nagai E, Tanaka M. MicroRNA-21 modulates biological functions of pancreatic cancer cells including their proliferation, invasion, and chemoresistance. Mol Cancer Ther. 2009;8(5):1067-74.

56. Zhang S, Hao J, Xie F, Hu X, Liu C, Tong J, Zhou J, Wu J, Shao C. Downregulation of miR-132 by promoter methylation contributes to pancreatic cancer development. Carcinogenesis. 2011;32(8):1183-9.

57. Tavano F, di Mola FF, Piepoli A, Panza A, Copetti M, Burbaci FP, Latiano T, Pellegrini F, Maiello E, Andriulli A, et al. Changes in miR-143 and miR-21 expression and clinicopathological correlations in pancreatic cancers. Pancreas. 2012;41(8):1280-4.

58. Niwa R, Slack FJ. The evolution of animal microRNA function. Curr Opin Genet Dev. 2007;17(2):145-50.

59. Papagiannakopoulos T, Shapiro A, Kosik KS. MicroRNA-21 targets a network of key tumor-suppressive pathways in glioblastoma cells. Cancer Res. 2008; 68(19):8164-72.

60. Tili E, Michaille JJ, Croce CM. MicroRNAs play a central role in molecular dysfunctions linking inflammation with cancer. Immunol Rev. 2013;253(1):167-84.

61. Piepoli A, Tavano F, Copetti M, Mazza T, Palumbo O, Panza A, di Mola FF, Pazienza V, Mazzoccoli G, Biscaglia G, et al. Mirna expression profiles identify drivers in colorectal and pancreatic cancers. PLoS One. 2012;7(3), e33663. 
62. Zhong Z, Dong Z, Yang L, Chen X, Gong Z. MicroRNA-31-5p modulates cell cycle by targeting human mutL homolog 1 in human cancer cells. Tumour Biol. 2013;34(3):1959-65.

63. Sun D, Yu F, Ma Y, Zhao R, Chen X, Zhu J, Zhang CY, Chen J, Zhang J. MicroRNA-31 activates the RAS pathway and functions as an oncogenic MicroRNA in human colorectal cancer by repressing RAS p21 GTPase activating protein 1 (RASA1). J Biol Chem. 2013;288(13):9508-18.

64. Xue Y, Abou Tayoun AN, Abo KM, Pipas JM, Gordon SR, Gardner TB, Barth Jr RJ, Suriawinata AA, Tsongalis GJ. MicroRNAs as diagnostic markers for pancreatic ductal adenocarcinoma and its precursor, pancreatic intraepithelial neoplasm. Cancer Genet. 2013;206(6):217-21.

65. Hong TH, Park IY. MicroRNA expression profiling of diagnostic needle aspirates from surgical pancreatic cancer specimens. Ann Surg Treat Res. 2014:87(6):290-7.

66. Zhao WG, Yu SN, Lu ZH, Ma YH, Gu YM, Chen J. The miR-217 microRNA functions as a potential tumor suppressor in pancreatic ductal adenocarcinoma by targeting KRAS. Carcinogenesis. 2010;31(10): 1726-33.

67. Deng S, Zhu S, Wang B, Li X, Liu Y, Qin Q, Gong Q, Niu Y, Xiang C, Chen J, et al. Chronic pancreatitis and pancreatic cancer demonstrate active epithelial-mesenchymal transition profile, regulated by miR-217-SIRT1 pathway. Cancer Lett. 2014;355(2):184-91.

68. Kalluri Sai Shiva UM, Kuruva MM, Mitnala S, Rupjyoti T, Guduru Venkat R, Botlagunta S, Kandagaddala R, Siddapuram SP, Sekaran A, Chemalakonda R, et al. MicroRNA profiling in periampullary carcinoma. Pancreatology. 2014; 14(1):36-47.

69. Gu J, Wang Y, Wu X. MicroRNA in the pathogenesis and prognosis of esophageal cancer. Curr Pharm Des. 2013;19(7):1292-300.

70. Ding L, Xu Y, Zhang W, Deng Y, Si M, Du Y, Yao H, Liu X, Ke Y, Si J, et al. MiR-375 frequently downregulated in gastric cancer inhibits cell proliferation by targeting JAK2. Cell Res. 2010;20(7):784-93.

71. Luo D, Wilson JM, Harvel N, Liu J, Pei L, Huang S, Hawthorn L, Shi H. A systematic evaluation of miRNA:mRNA interactions involved in the migration and invasion of breast cancer cells. J Transl Med. 2013;11:57.

72. Li Y, Jiang Q, Xia N, Yang H, Hu C. Decreased expression of microRNA-375 in nonsmall cell lung cancer and its clinical significance. J Int Med Res. 2012; 40(5):1662-9.

73. Dai X, Chiang Y, Wang Z, Song Y, Lu C, Gao P, Xu H. Expression levels of microRNA-375 in colorectal carcinoma. Mol Med Rep. 2012;5(5):1299-304.

74. Bierkens M, Krijgsman O, Wilting SM, Bosch L, Jaspers A, Meijer GA, Meijer CJ, Snijders PJ, Ylstra B, Steenbergen RD. Focal aberrations indicate EYA2 and hsa-miR-375 as oncogene and tumor suppressor in cervical carcinogenesis. Genes Chromosomes Cancer. 2013;52(1):56-68.

75. Poy MN, Hausser J, Trajkovski M, Braun M, Collins S, Rorsman P, Zavolan M, Stoffel M. miR-375 maintains normal pancreatic alpha- and beta-cell mass. Proc Natl Acad Sci U S A. 2009;106(14):5813-8.

76. Zhao H, Guan J, Lee HM, Sui Y, He L, Siu JJ, Tse PP, Tong PC, Lai FM, Chan JC. Up-regulated pancreatic tissue microRNA-375 associates with human type 2 diabetes through beta-cell deficit and islet amyloid deposition. Pancreas. 2010;39(6):843-6.

77. Zhou J, Song S, Cen J, Zhu D, Li D, Zhang Z. MicroRNA-375 is downregulated in pancreatic cancer and inhibits cell proliferation in vitro. Oncol Res. 2012;20(5-6):197-203.

78. Song SD, Zhou J, Zhou J, Zhao H, Cen JN, Li DC. MicroRNA-375 targets the 3-phosphoinositide-dependent protein kinase-1 gene in pancreatic carcinoma. Oncol Lett. 2013;6(4):953-9.

79. Ueda T, Volinia S, Okumura H, Shimizu M, Taccioli C, Rossi S, Alder H, Liu CG, Oue N, Yasui W, et al. Relation between microRNA expression and progression and prognosis of gastric cancer: a microRNA expression analysis. Lancet Oncol. 2010;11(2):136-46.

80. Chen KJ, Hou Y, Wang K, Li J, Xia Y, Yang XY, LV G, Xing XL, Shen F. Reexpression of Let-7 $\mathrm{g}$ microRNA inhibits the proliferation and migration via K-Ras/HMGA2/snail axis in hepatocellular carcinoma. Biomed Res Int. 2014:2014:742417.

81. Lu L, Katsaros D, Shaverdashvili K, Qian B, Wu Y, de la Longrais IA, Preti M, Menato G, Yu H. Pluripotent factor lin-28 and its homologue lin-28b in epithelial ovarian cancer and their associations with disease outcomes and expression of let-7a and IGF-II. Eur J Cancer. 2009:45(12):2212-8.

82. Wang S, Tang Y, Cui H, Zhao X, Luo X, Pan W, Huang X, Shen N. Let-7/miR-98 regulate Fas and Fas-mediated apoptosis. Genes Immun. 2011;12(2):149-54
83. Kang W, Tong JH, Lung RW, Dong Y, Yang W, Pan Y, Lau KM, Yu J, Cheng AS, To KF. let-7b/g silencing activates AKT signaling to promote gastric carcinogenesis. J Transl Med. 2014;12:281.

84. Hu X, Guo J, Zheng L, Li C, Zheng TM, Tanyi JL, Liang S, Benedetto C, Mitidieri M, Katsaros D, et al. The heterochronic microRNA let-7 inhibits cell motility by regulating the genes in the actin cytoskeleton pathway in breast cancer. Mol Cancer Res. 2013;11(3):240-50.

85. Lan FF, Wang H, Chen YC, Chan CY, Ng SS, Li K, Xie D, He ML, Lin MC, Kung HF. Hsa-let-7 $\mathrm{g}$ inhibits proliferation of hepatocellular carcinoma cells by downregulation of c-Myc and upregulation of p16(INK4A). Int J Cancer. 2011;128(2):319-31.

86. Nagano H, Tomimaru $Y$, Eguchi H, Hama N, Wada H, Kawamoto K, Kobayashi S, Mori M, Doki Y. MicroRNA-29a induces resistance to gemcitabine through the Wnt/beta-catenin signaling pathway in pancreatic cancer cells. Int J Oncol. 2013;43(4):1066-72.

87. Chen J, Li Q, An Y, Lv N, Xue X, Wei J, Jiang K, Wu J, Gao W, Qian Z, et al. CEACAM6 induces epithelial-mesenchymal transition and mediates invasion and metastasis in pancreatic cancer. Int J Oncol. 2013;43(3):877-85.

88. Di Leva G, Garofalo M, Croce CM. MicroRNAs in cancer. Annu Rev Pathol. 2014;9:287-314

89. Yang S, Li Y, Gao J, Zhang T, Li S, Luo A, Chen H, Ding F, Wang X, Liu Z. MicroRNA-34 suppresses breast cancer invasion and metastasis by directly targeting Fra-1. Oncogene. 2012.

90. Ali S, Ahmad A, Aboukameel A, Ahmed A, Bao B, Banerjee S, Philip PA, Sarkar FH. Deregulation of miR-146a expression in a mouse model of pancreatic cancer affecting EGFR signaling. Cancer Lett. 2014;351(1):134-42.

91. Gregory PA, Bracken CP, Bert AG, Goodall GJ. MicroRNAs as regulators of epithelial-mesenchymal transition. Cell Cycle. 2008;7(20):3112-8.

92. Verdoodt B, Neid M, Vogt M, Kuhn V, Liffers ST, Palisaar RJ, Noldus J, Tannapfel A, Mirmohammadsadegh A. MicroRNA-205, a novel regulator of the anti-apoptotic protein $\mathrm{BCl} 2$, is downregulated in prostate cancer. Int J Oncol. 2013;43(1):307-14.

93. Iorio MV, Casalini P, Piovan C, Di Leva G, Merlo A, Triulzi T, Menard S, Croce CM, Tagliabue E. microRNA-205 regulates HER3 in human breast cancer. Cancer Res. 2009;69(6):2195-200.

94. Jiang L, Huang Q, Chang J, Wang E, Qiu X. MicroRNA HSA-miR-125a-5p induces apoptosis by activating p53 in lung cancer cells. Exp Lung Res. 2011;37(7):387-98

95. Ma Y, Zhang P, Yang J, Liu Z, Yang Z, Qin H. Candidate microRNA biomarkers in human colorectal cancer: systematic review profiling studies and experimental validation. Int J Cancer. 2012;130(9):2077-87.

\section{Submit your next manuscript to BioMed Central and we will help you at every step:}

- We accept pre-submission inquiries

- Our selector tool helps you to find the most relevant journal

- We provide round the clock customer support

- Convenient online submission

- Thorough peer review

- Inclusion in PubMed and all major indexing services

- Maximum visibility for your research

Submit your manuscript at www.biomedcentral.com/submit
Biomed Central 